\title{
Safety and Effectiveness of Ipragliflozin for Type 2 Diabetes in Japan: 12-Month Interim Results of the STELLA-LONG TERM Post-Marketing Surveillance Study
}

\author{
Ichiro Nakamura · Hiroshi Maegawa $\cdot$ Kazuyuki Tobe • \\ Satoshi Uno
}

Received: October 19, 2018 / Published online: February 14, 2019

(C) The Author(s) 2019

\section{ABSTRACT}

Introduction: The present interim report of the STELLA-LONG TERM study aimed to examine the safety and effectiveness of ipragliflozin in real-word clinical practice in Japan using data up to 12 months. We also evaluated the effect of ipragliflozin on aspartate aminotransferase (AST) and alanine aminotransferase (ALT) levels in patients with normal vs. abnormal liver function.

Methods: This is an ongoing 3-year post-marketing surveillance study. We analyzed data from Japanese type 2 diabetes mellitus (T2DM) patients who were first prescribed ipragliflozin between 17 July 2014 and 16 October 2015 at participating centers in Japan, and whose data were locked by 16 January 2018 . The incidence of adverse drug reactions (ADRs) was evaluated for safety. Changes in glycemic control and

Enhanced Digital Features To view enhanced digital features for this article go to https://doi.org/10.6084/ m9.figshare.7629557.

I. Nakamura $(\bowtie) \cdot S$. Uno

Astellas Pharma Inc., Tokyo, Japan

e-mail: ichiro.nakamura@astellas.com

H. Maegawa

Department of Medicine, Shiga University of

Medical Science, Shiga, Japan

K. Tobe

First Department of Internal Medicine, University of

Toyama, Toyama, Japan body weight were evaluated for effectiveness. The effect on liver function was evaluated by changes in the fatty liver index, and changes in AST and ALT were evaluated in patients with normal and abnormal liver function.

Results: The safety analysis set comprised 11,051 patients and the efficacy analysis set comprised 8788 patients. The incidence rates of ADRs and serious ADRs were 14.6\% (1616/ $11,051)$ and $0.97 \%(107 / 11,051)$, respectively. Significant reductions (all $P<0.001$ vs. baseline, paired $t$ test) in glycated hemoglobin $(-0.8 \%)$, fasting plasma glucose $(-31.9 \mathrm{mg} / \mathrm{dL})$, body weight $(-2.9 \mathrm{~kg})$, and fatty liver index $(-8.7)$ were observed. In patients with normal liver function at baseline, no clinically significant changes in AST and ALT were observed. In patients with abnormal liver function at baseline, clinically and statistically significant decreases $(P<0.05$ vs. baseline, two-sample $t$ test) in AST $(-9.0 \mathrm{U} / \mathrm{L})$ and ALT $(-14.7 \mathrm{U} / \mathrm{L})$ levels were observed.

Conclusion: Ipragliflozin was effective and well tolerated in Japanese patients with T2DM over 12 months in the real-world clinical setting. Improvements in liver function parameters (AST and ALT) were observed in T2DM patients with abnormal liver function.

Trial Registration: ClinicalTrials.gov identifier, NCT02479399.

Funding: Astellas Pharma Inc., Japan. 
Keywords: Effectiveness; Ipragliflozin; Japan; Post-marketing surveillance; Safety; Sodiumglucose cotransporter 2 inhibitor; Type 2 diabetes mellitus

\section{INTRODUCTION}

Ipragliflozin is a sodium-glucose cotransporter 2 (SGLT2) inhibitor that was approved in Japan in January 2014 for the treatment of type 2 diabetes mellitus (T2DM) patients [1]. Although SGLT2 inhibitors are known to provide benefits in relation to the low risk of hypoglycemia and promotion of weight loss [2], there are limited data on the safety and effectiveness of the SGLT2 inhibitor ipragliflozin in the real-world setting in Japan.

Several post-marketing surveillance studies of ipragliflozin have been conducted under the guidance of the Japanese Pharmaceuticals and Medical Devices Agency. The STELLA-LONG TERM study is an ongoing 3-year post-marketing surveillance study of ipragliflozin in Japanese patients with T2DM in real-world clinical practice $[3,4]$. Subgroup analyses based on the interim cutoff data have been conducted to evaluate the long-term safety and effectiveness of ipragliflozin in elderly vs. non-elderly patients and stratified by body mass index (BMI) and liver function status (normal vs. abnormal) $[5,6]$. Recently published interim reports of the STELLA-LONG TERM study included a pooled analysis of 3-, 12-, and 24-month data [4, 5]. The results of the analyses conducted thus far have shown that ipragliflozin was effective in improving glycemic control without raising new safety concerns in T2DM patients [3, 4], regardless of age [5] or BMI [data on file], and improved liver function in T2DM patients with abnormal liver function [6].

Preclinical study findings have suggested an improvement in liver function with SGLT2 inhibitors [7-16], and a pooled analysis of five randomized controlled trials showed improvements in both aspartate aminotransferase (AST) and alanine aminotransferase (ALT) levels with the use of ipragliflozin [17]. However, the potential effects of ipragliflozin on liver function-related parameters in T2DM patients, especially in those with abnormal liver function, are poorly understood and warrant further investigation.

The present interim report of the STELLALONG TERM study aimed to examine the safety and effectiveness of ipragliflozin in real-world clinical practice in Japan using data from patients up to 12 months of follow-up. We also evaluated the effect of ipragliflozin on AST and ALT levels in patients with normal vs. abnormal liver function.

\section{METHODS}

\section{Study Design and Patients}

This is an ongoing 3-year post-marketing surveillance study. Details of the study design, patient selection criteria, and methods are described in the first interim report [3]. This study was performed in compliance with Good Post-marketing Study Practice. Anonymized data were collected from a clinical setting as required by the regulatory authority; therefore, informed consent was waived in compliance with the policies of each study site. All of the medical institutions that agreed to provide data signed a contract with Astellas Pharma Inc.

Data from T2DM patients who were first prescribed ipragliflozin between 17 July 2014 and 16 October 2015 at one of the 2431 participating centers in Japan were recorded in electronic case report forms, collected in an electronic database, and analyzed. Baseline and post-baseline data on the following survey items were collected: demographic characteristics; body weight and blood pressure; duration of diabetes; complications; liver function status; use of concomitant antidiabetic drugs, diuretics, and other drugs; laboratory data [glycated hemoglobin (HbA1c), fasting plasma glucose, estimated glomerular filtration rate (eGFR), fasting serum insulin, AST, and ALT]; and safety data.

Further details on how the survey was conducted have been described previously [3]. The cutoff date for the present 12-month interim report was 16 January 2018. 


\section{Treatment}

A once-daily dose of ipragliflozin $50 \mathrm{mg}$ was administered before or after breakfast, in accordance with the package insert. In patients with severe hepatic impairment, a lower dose was allowed at the discretion of the attending physician. If the attending physician considered the treatment effectiveness as insufficient, a dose and ALT were evaluated in patients with normal and abnormal liver function. Patients with normal liver function were defined as those with baseline ALT $\leq 30 \mathrm{U} / \mathrm{L}$ (male) or $\leq 20 \mathrm{U} / \mathrm{L}$ (female). Patients with abnormal liver function were defined as those with baseline ALT $\geq 31 \mathrm{U} /$ $\mathrm{L}$ (male) or $\geq 21 \mathrm{U} / \mathrm{L}$ (female). The fatty liver index was calculated in accordance with an algorithm proposed by Bedogni et al. [18]:

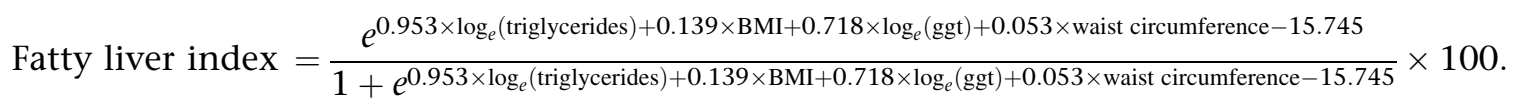

increase to $100 \mathrm{mg}$ was allowed with careful monitoring of the patient's clinical course.

\section{Vital Signs and Laboratory Variables}

Changes in vital signs (including systolic and diastolic blood pressure), laboratory parameters, and eGFR were evaluated.

\section{Safety}

Adverse drug reactions (ADRs) were evaluated and categorized by system organ class (SOC) and preferred term using the Medical Dictionary for Regulatory Activities (MedDRA)/J version 20.1. The time to onset of ADRs and continuation/discontinuation of ipragliflozin treatment associated with ADRs were also evaluated.

\section{Effectiveness}

Effectiveness outcome measures were changes in glycemic control (HbA1c, fasting plasma glucose, and fasting serum insulin) and body weight.

\section{Liver Function}

Liver function assessments included changes in AST, ALT, and fatty liver index. Changes in AST
Hepatic steatosis is indicated by a fatty liver index $\geq 60$ and ruled out by a fatty liver index $<30$. The correlation between changes in ALT and other parameters related to glycemic control and blood pressure, among others, was evaluated in patients with abnormal liver function.

\section{Statistical Analysis}

The rationale for the study length and sample size calculations have been described previously [3]. No sample size calculation was performed for subgroup comparisons of patients with normal and abnormal liver function. The safety and efficacy analysis sets included all patients observed for up to 12 months. Categorical variables are shown as $n(\%)$ and continuous variables are shown as mean \pm standard deviation (SD), unless otherwise specified. Paired $t$ tests were used to evaluate changes in laboratory parameters from baseline to $1,3,6$, and 12 months. Two-sample $t$ tests were used for comparisons of liver function tests between patients with normal and abnormal liver function. Statistical significance was set at two-sided $P<0.05$. Adjustments for type I error, based on multiple hypothesis testing, were not performed. In patients with abnormal liver function, Pearson's correlation coefficient and its $P$ value were calculated to evaluate the relationship between changes in ALT and changes 
in other parameters. All statistical analyses were performed using SAS statistical software version 9.3 (SAS Institute Inc., Cary, NC, USA) or higher.

\section{RESULTS}

\section{Patient Disposition}

The disposition of patients is shown in Fig. 1. Of 2431 institutions that agreed to participate in this study, 1941 participated and initially registered 11,424 patients. Survey forms were collected for 9991 patients at 12 months. Out of 11,289 patients included in the locked database, the safety analysis set comprised 11,051 patients at 3 months and 9970 patients at 12 months. Among the 11,051 patients, 2263 patients were excluded from the efficacy analysis set, which subsequently comprised 8788 patients. Patients were excluded mainly because of noncompliance with the study drug (e.g., starting dose other than $50 \mathrm{mg}$ once daily for patients without severe hepatic impairment); unclear effectiveness assessment; or no effectiveness data available for $\mathrm{HbA1c}$, serum fasting insulin or fasting plasma glucose at baseline or post-baseline.

\section{Patient Characteristics}

The baseline demographic and clinical characteristics of patients are shown in Table 1 . Of the 11,051 patients in the safety analysis set, 6712 $(60.7 \%)$ were male. In the safety analysis set, the mean \pm SD age was $56.9 \pm 12.2$ years, BMI was $29.1 \pm 5.3 \mathrm{~kg} / \mathrm{m}^{2}$, and duration of diabetes was $8.0 \pm 6.5$ years. Treatments used at baseline and during the survey period are shown in Table 2. Most patients (81.5\%) were receiving treatment with concomitant antidiabetic drugs, among which the most common types were dipeptidyl peptidase-4 (DPP-4) inhibitors (56.3\%),

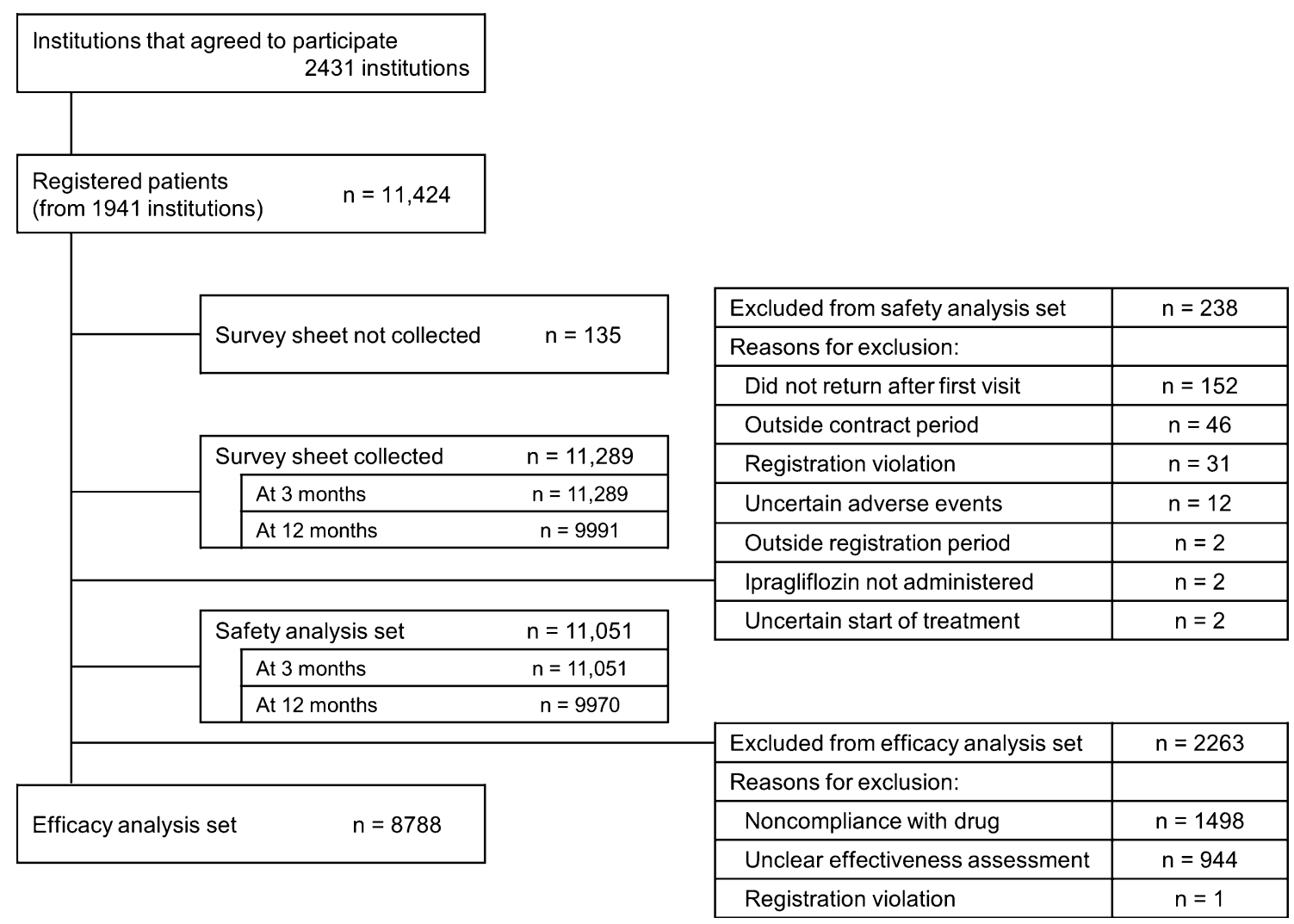

Fig. 1 Patient disposition 
Table 1 Patient characteristics at baseline

\begin{tabular}{|c|c|c|}
\hline & \multicolumn{2}{|c|}{ Number of patients $(\%)$ or mean $\pm S D$} \\
\hline & Safety analysis set & $\overline{\text { Efficacy analysis set }}$ \\
\hline Total & $11,051(100.0)$ & $8788(100.0)$ \\
\hline \multicolumn{3}{|l|}{ Sex } \\
\hline Male & $6712(60.7)$ & $5384(61.3)$ \\
\hline Female & $4339(39.3)$ & $3404(38.7)$ \\
\hline \multicolumn{3}{|l|}{ Age, years } \\
\hline \multirow[t]{2}{*}{$n$} & 11,051 & 8788 \\
\hline & $56.9 \pm 12.2$ & $56.4 \pm 12.0$ \\
\hline \multicolumn{3}{|l|}{ Body weight, kg } \\
\hline \multirow[t]{2}{*}{$n$} & 8170 & 6901 \\
\hline & $78.1 \pm 17.3$ & $78.5 \pm 17.2$ \\
\hline \multicolumn{3}{|l|}{ BMI, $\mathrm{kg} / \mathrm{m}^{2}$} \\
\hline \multirow[t]{2}{*}{$n$} & 7489 & 6336 \\
\hline & $29.1 \pm 5.3$ & $29.2 \pm 5.3$ \\
\hline \multicolumn{3}{|l|}{ Inpatient/outpatient } \\
\hline Inpatient & $183(1.7)$ & $153(1.7)$ \\
\hline Outpatient & $10,868(98.3)$ & $8635(98.3)$ \\
\hline \multicolumn{3}{|l|}{ Duration of diabetes, years } \\
\hline \multirow[t]{2}{*}{$n$} & 7247 & 5942 \\
\hline & $8.0 \pm 6.5$ & $8.0 \pm 6.4$ \\
\hline \multicolumn{3}{|l|}{ Duration of diabetes, category } \\
\hline$<5$ years & $2593(23.5)$ & $2116(24.1)$ \\
\hline$\geq 5$ years to $<10$ years & $2171(19.6)$ & $1775(20.2)$ \\
\hline$\geq 10$ years to $<15$ years & $1425(12.9)$ & $1179(13.4)$ \\
\hline$\geq 15$ years & $1058(9.6)$ & $872(9.9)$ \\
\hline Unknown & $3804(34.4)$ & $2846(32.4)$ \\
\hline \multicolumn{3}{|l|}{ Complications } \\
\hline No & $1637(14.8)$ & $1293(14.7)$ \\
\hline Yes & $9321(84.3)$ & $7443(84.7)$ \\
\hline Unknown & $93(0.8)$ & $52(0.6)$ \\
\hline \multicolumn{3}{|c|}{ Type of complication (some patients had more than one complication) } \\
\hline Diabetic neuropathy & $956(8.7)$ & $787(9.0)$ \\
\hline Diabetic nephropathy & $1819(16.5)$ & $1516(17.3)$ \\
\hline
\end{tabular}


Table 1 continued

\begin{tabular}{|c|c|c|}
\hline & \multicolumn{2}{|c|}{ Number of patients (\%) or mean $\pm S D$} \\
\hline & Safety analysis set & $\overline{\text { Efficacy analysis set }}$ \\
\hline Diabetic retinopathy & $884(8.0)$ & $741(8.4)$ \\
\hline Cardiovascular and cerebrovascular disease & $1027(9.3)$ & $748(8.5)$ \\
\hline Myocardial infarction & $146(1.3)$ & $118(1.3)$ \\
\hline Angina pectoris & $467(4.2)$ & $323(3.7)$ \\
\hline Heart failure & $230(2.1)$ & $161(1.8)$ \\
\hline Arteriosclerosis obliterans & $139(1.3)$ & $99(1.1)$ \\
\hline Cerebrovascular disease & $261(2.4)$ & $201(2.3)$ \\
\hline Hypertension & $6183(55.9)$ & $4921(56.0)$ \\
\hline Dyslipidemia (hyperlipidemia) & $7055(63.8)$ & $5657(64.4)$ \\
\hline Osteoporosis & $184(1.7)$ & $142(1.6)$ \\
\hline Hyperuricemia & $1042(9.4)$ & $812(9.2)$ \\
\hline Urinary tract infection & $21(0.2)$ & $18(0.2)$ \\
\hline Genital infection & $8(0.1)$ & $6(0.1)$ \\
\hline Malignant tumor & $79(0.7)$ & $62(0.7)$ \\
\hline Other & $4050(36.6)$ & $3264(37.1)$ \\
\hline \multicolumn{3}{|l|}{ Hepatic function status* } \\
\hline Normal & $8320(75.3)$ & $6525(74.2)$ \\
\hline Mild impairment & $2110(19.1)$ & $1761(20.0)$ \\
\hline Moderate impairment & $299(2.7)$ & $252(2.9)$ \\
\hline Severe impairment & $10(0.1)$ & $9(0.1)$ \\
\hline Unknown & $312(2.8)$ & $241(2.7)$ \\
\hline \multicolumn{3}{|l|}{ Renal function status* } \\
\hline Normal & $9215(83.4)$ & $7338(83.7)$ \\
\hline Mild impairment & $1396(12.6)$ & $1110(12.6)$ \\
\hline Moderate impairment & $125(1.1)$ & $98(1.1)$ \\
\hline Severe impairment & $10(0.1)$ & $7(0.1)$ \\
\hline Unknown & $305(2.8)$ & $235(2.7)$ \\
\hline \multicolumn{3}{|l|}{$\mathrm{eGFR}, \mathrm{mL} / \mathrm{min} / 1.73 \mathrm{~m}^{2}$} \\
\hline \multirow[t]{2}{*}{$n$} & 6757 & 5682 \\
\hline & $82.1 \pm 21.7$ & $82.4 \pm 21.5$ \\
\hline
\end{tabular}


Table 1 continued

\begin{tabular}{lll}
\hline & \multicolumn{2}{l}{ Number of patients (\%) or mean \pm SD } \\
\cline { 2 - 3 } & Safety analysis set & Efficacy analysis set \\
\hline eGFR, category, $\mathrm{mL} / \mathrm{min} / 1.73 \mathrm{~m}^{2}$ & & $1841(20.9)$ \\
$\geq 90$ & $2162(19.6)$ & $3117(35.5)$ \\
$\geq 60$ to $<90$ & $3685(33.3)$ & $582(6.6)$ \\
$\geq 45$ to $<60$ & $722(6.5)$ & $120(1.4)$ \\
$\geq 30$ to $<45$ & $155(1.4)$ & $22(0.3)$ \\
$<30$ & $33(0.3)$ & $3106(35.3)$ \\
Unknown & $4294(38.9)$ & \\
\hline
\end{tabular}

*Judged by the attending physician using the classification criteria for seriousness of adverse drug reactions of pharmaceuticals [33]

$B M I$ body mass index, $e G F R$ estimated glomerular filtration rate, $S D$ standard deviation

metformin $(42.3 \%)$, and sulfonylureas (28.2\%). Only $7.6 \%$ of patients were receiving concomitant diuretics.

\section{Vital Signs}

The changes from baseline in vital signs are shown in Table 3 . Statistically significant decreases (all $P<0.05$ ) were observed in systolic blood pressure $(-4.3 \pm 14.6 \mathrm{mmHg})$, diastolic blood pressure $(-2.6 \pm 10.0 \mathrm{mmHg})$, and heart rate $(-0.9 \pm 9.4$ beats $/ \mathrm{min})$ from baseline to 12 months.

\section{Laboratory Variables}

The changes from baseline in laboratory parameters are shown in Table 3. Significant decreases (both $P<0.05$ ) were observed in AST $(-4.6 \pm 16.3 \mathrm{U} / \mathrm{L})$ and ALT $(-7.9 \pm 21.1 \mathrm{U} / \mathrm{L})$ levels from baseline to 12 months. Mean changes in total cholesterol $(-2.5 \pm 30.7 \mathrm{mg} / \mathrm{dL})$, low-density lipoprotein cholesterol $(-4.0 \pm$ $26.7 \mathrm{mg} / \mathrm{dL})$, and triglyceride levels $(-16.3 \pm$ $143.2 \mathrm{mg} / \mathrm{dL}$ ) showed significant decreases (all $P<0.05)$, and mean changes in high-density lipoprotein cholesterol $(2.9 \pm 9.2 \mathrm{mg} / \mathrm{dL})$ showed a significant increase from baseline to 12 months. Mean changes in serum uric acid concentration $\quad(-0.29 \pm 0.95 \mathrm{mg} / \mathrm{dL})$ also showed a significant decrease $(P<0.05)$ from baseline to 12 months. Mean changes in hematocrit $(1.9 \pm 2.8 \%)$ and blood urea nitrogen $(1.2 \pm 3.7 \mathrm{mg} / \mathrm{dL})$ showed a significant increase $(P<0.05)$ from baseline to 12 months. Changes in eGFR from baseline are shown in Fig. 2a. The eGFR decreased significantly $\left(-2.3 \pm 11.2 \mathrm{~mL} / \mathrm{min} / 1.73 \mathrm{~m}^{2}\right)$ at $12 \mathrm{months}$ from a baseline value of $82.1 \pm 21.7 \mathrm{~mL} / \mathrm{min} /$ $1.73 \mathrm{~m}^{2}(P<0.001)$.

\section{Safety}

Safety was assessed in 11,051 patients in the safety analysis set. ADRs are listed in Table 4 by SOC, together with the rates of these ADRs in clinical trials prior to ipragliflozin approval [19-24]. Overall, 2239 ADRs in 1616 patients were reported with an incidence rate of $14.6 \%$. The most common class of ADRs was renal and urinary disorders, which occurred in 644 patients $(5.8 \%)$, followed by investigations in 205 patients $(1.9 \%)$, infections and infestations in 201 patients $(1.8 \%)$, metabolism and nutrition disorders in 161 patients (1.5\%), and skin and subcutaneous tissue disorders in 147 patients (1.3\%). All other classes of ADRs occurred in less than $1 \%$ of patients. In the present survey, no new unreported safety concerns were observed. 
Table 2 Treatments used at baseline and during the survey period

\begin{tabular}{|c|c|c|}
\hline & \multicolumn{2}{|c|}{ Number of patients $(\%)$ or mean $\pm S D$} \\
\hline & Safety analysis set & Efficacy analysis set \\
\hline \multicolumn{3}{|l|}{ Total } \\
\hline- & $11,051(100.0)$ & $8788(100.0)$ \\
\hline \multicolumn{3}{|c|}{ Initial dose of ipragliflozin, mg } \\
\hline 25 & $1421(12.9)$ & 0 \\
\hline 50 & $9612(87.0)$ & $8788(100.0)$ \\
\hline 75 & 0 & 0 \\
\hline 100 & $14(0.1)$ & 0 \\
\hline Other & $4(0.0)$ & 0 \\
\hline \multicolumn{3}{|c|}{ Daily dose of ipragliflozin, mg } \\
\hline \multirow[t]{2}{*}{$n$} & 11,051 & 8788 \\
\hline & $47.5 \pm 8.5$ & $50.3 \pm 2.8$ \\
\hline \multicolumn{3}{|c|}{ Concomitant treatment } \\
\hline \multicolumn{3}{|c|}{ Concomitant antidiabetic drugs } \\
\hline No & $1964(17.8)$ & $1489(16.9)$ \\
\hline Yes & $9006(81.5)$ & $7248(82.5)$ \\
\hline Unknown & $81(0.7)$ & $51(0.6)$ \\
\hline \multicolumn{3}{|c|}{ Number of concomitant antidiabetic drugs } \\
\hline \multirow[t]{2}{*}{$n$} & 11,051 & 8788 \\
\hline & $1.6 \pm 1.2$ & $1.6 \pm 1.2$ \\
\hline $\operatorname{Max}$ & 6 & 6 \\
\hline Min & 0 & 0 \\
\hline 0 & $2189(19.8)$ & $1680(19.1)$ \\
\hline 1 & $3129(28.3)$ & $2441(27.8)$ \\
\hline 2 & $3195(28.9)$ & $2613(29.7)$ \\
\hline 3 & $1837(16.6)$ & $1487(16.9)$ \\
\hline$\geq 4$ & $619(5.6)$ & $515(5.9)$ \\
\hline Unknown & $82(0.7)$ & $52(0.6)$ \\
\hline \multicolumn{3}{|c|}{ Type of concomitant antidiabetic drugs (some patients had more than one concomitant drug) } \\
\hline DPP-4 inhibitor & $6222(56.3)$ & $5028(57.2)$ \\
\hline Metformin & $4670(42.3)$ & $3891(44.3)$ \\
\hline Sulfonylurea & $3117(28.2)$ & $2474(28.2)$ \\
\hline Insulin injection & $1213(11.0)$ & $1003(11.4)$ \\
\hline
\end{tabular}


Table 2 continued

\begin{tabular}{|c|c|c|}
\hline \multicolumn{3}{|c|}{ Number of patients (\%) or mean \pm SD } \\
\hline & Safety analysis set & Efficacy analysis set \\
\hline$\alpha$-Glucosidase inhibitor & $1150(10.4)$ & $918(10.4)$ \\
\hline Thiazolidinedione & $949(8.6)$ & $774(8.8)$ \\
\hline GLP-1 receptor agonist & $380(3.4)$ & $333(3.8)$ \\
\hline Fast-acting insulin secretagogue & $340(3.1)$ & $279(3.2)$ \\
\hline Others & $840(7.6)$ & $657(7.5)$ \\
\hline \multicolumn{3}{|l|}{ Concomitant diuretics } \\
\hline No & $10,108(91.5)$ & $8065(91.8)$ \\
\hline Yes & $839(7.6)$ & $643(7.3)$ \\
\hline Unknown & $104(0.9)$ & $80(0.9)$ \\
\hline \multicolumn{3}{|c|}{ Type of concomitant diuretics (some patients had more than one concomitant drug) } \\
\hline Thiazide diuretic & $215(1.9)$ & $168(1.9)$ \\
\hline Loop diuretic & $204(1.8)$ & $151(1.7)$ \\
\hline Potassium-sparing diuretic & $172(1.6)$ & $133(1.5)$ \\
\hline Vasopressin antagonist & $6(0.1)$ & $5(0.1)$ \\
\hline Osmotic diuretic & $1(0.0)$ & 0 \\
\hline Carbonate dehydratase inhibitor & 0 & 0 \\
\hline Others & $365(3.3)$ & $287(3.3)$ \\
\hline \multicolumn{3}{|l|}{ Other concomitant drugs } \\
\hline No & $3051(27.6)$ & $2436(27.7)$ \\
\hline Yes & $7876(71.3)$ & $6275(71.4)$ \\
\hline Unknown & $124(1.1)$ & $77(0.9)$ \\
\hline \multicolumn{3}{|c|}{ Type of other concomitant drugs (some patients had more than one concomitant drug) } \\
\hline Antihypertensive drug & $4934(44.6)$ & $3923(44.6)$ \\
\hline $\mathrm{ARB}$ & $2519(22.8)$ & $2019(23.0)$ \\
\hline $\mathrm{CCB}$ & $2306(20.9)$ & $1790(20.4)$ \\
\hline $\mathrm{ARB}+\mathrm{CCB}$ & $1373(12.4)$ & $1107(12.6)$ \\
\hline Statin & $4151(37.6)$ & $3280(37.3)$ \\
\hline Antiplatelet drug & $974(8.8)$ & $737(8.4)$ \\
\hline Antipeptic ulcer drug & $989(8.9)$ & $790(9.0)$ \\
\hline Antihyperuricemic drug & $726(6.6)$ & $564(6.4)$ \\
\hline
\end{tabular}


Table 2 continued

\begin{tabular}{lll}
\hline & \multicolumn{2}{l}{ Number of patients (\%) or mean $\mathbf{I}$ SD } \\
\cline { 2 - 3 } & Safety analysis set & Efficacy analysis set \\
\hline Others & $3801(34.4)$ & $3002(34.2)$ \\
\hline
\end{tabular}

$A R B$ angiotensin receptor blocker, $C C B$ calcium channel blocker, DPP-4 dipeptidyl peptidase-4, GLP-1 glucagon-like peptide-1, SD standard deviation

Serious ADRs reported during the survey period are shown in Table 5. Overall, serious ADRs were observed in 107 patients (0.97\%). These included nervous system disorders (21 cases in total: cerebral infarction, 11; lacunar infarction, 3; cerebral hemorrhage, 2; depressed level of consciousness, dizziness, somnolence, transient ischemic attack, and putamen hemorrhage, 1 each); neoplasms benign, malignant, and unspecified (20 cases in total: pancreatic carcinoma, 6; colon cancer, 5; breast cancer female, 2; metastases to lymph nodes, pancreatic carcinoma metastatic, squamous cell carcinoma of skin, lung neoplasm malignant, prostate cancer, lung neoplasm, thyroid cancer, and intraductal proliferative breast lesion, 1 each); cardiac disorders (19 cases in total: acute myocardial infarction and myocardial infarction, 5 each; angina unstable, 3 ; angina pectoris and cardiac failure congestive, 2 each; atrial fibrillation, cardiovascular disorder, and coronary artery disease, 1 each); infections and infestations ( 9 cases in total: urinary tract infection, 3; pyelonephritis acute, 2; Escherichia sepsis, liver abscess, peritonsillar abscess, pneumonia, and genital herpes simplex, 1 each); hepatobiliary disorders ( 7 cases in total: cholecystitis and hepatic cirrhosis, 2 each; cholecystitis acute, cholelithiasis, jaundice cholestatic, and liver disorder, 1 each); metabolism and nutrition disorders ( 6 cases in total: dehydration and hypoglycemia, 2 each; hyperglycemia and hyperphagia, 1 each); renal and urinary disorders (6 cases in total: renal disorder, 2; hematuria, neurogenic bladder, renal vessel disorder, and ureterolithiasis, 1 each); skin and subcutaneous tissue disorders (4 cases in total: drug eruption, eczema, skin ulcer, and urticaria, 1 each); injury, poisoning, and procedural complications (4 cases in total: fall, femoral neck fracture, foot fracture, ligament sprain; road traffic accident; subarachnoid hemorrhage; contusion and brain contusion, 1 each); eye disorders (3 cases in total: retinal hemorrhage, 2; diabetic retinopathy, 1); vascular disorders (3 cases in total: hypertension, 2; varicose vein, 1 ); gastrointestinal disorders ( 3 cases in total: duodenal ulcer, gastric ulcer hemorrhage, gastroesophageal reflux disease, and pancreatitis acute, 1 each); psychiatric disorders (2 cases of depression); endocrine disorders (1 case of hypothyroidism); ear and labyrinth disorders (1 case of sudden hearing loss); reproductive system and breast disorders (1 case of prostatitis); general disorders and administration site conditions ( 1 case of thirst); and investigations (1 case of gamma-glutamyl transferase increased).

ADRs of special interest are shown in Table 6. The incidence of these ranged from $0.02 \%$ (fracture) to $5.2 \%$ (polyuria/pollakiuria). Although 571 patients (5.2\%) reported polyuria/pollakiuria, only one of these events was considered serious. These included 438 pollakiuria ADRs (4.0\%) and 293 polyuria ADRs (2.7\%). Volume depletion-related events, including dehydration, were reported in 196 patients $(1.8 \%)$, and nine $(0.08 \%)$ events were considered serious. ADRs associated with volume depletion included pollakiuria (26 ADRs, $0.24 \%$ ), polyuria (16 ADRs, $0.14 \%$ ), thirst (16 ADRs, $0.14 \%$ ), cerebral infarction (three ADRs, $0.03 \%$ ), and lacunar infarction (one ADR, $0.01 \%)$. Skin complications were reported in 166 patients $(1.5 \%)$, of which four $(0.04 \%)$ cases were considered serious. Skin complication-related ADRs included drug eruption (42 ADRs, $0.38 \%$ ), pruritus (34 ADRs, $0.31 \%$ ), pruritus genital (25 ADRs, 0.23\%), rash (18 ADRs, $0.16 \%$ ), and eczema (17 ADRs, 0.15\%). Genital infection was reported in 135 patients (1.2\%), of 


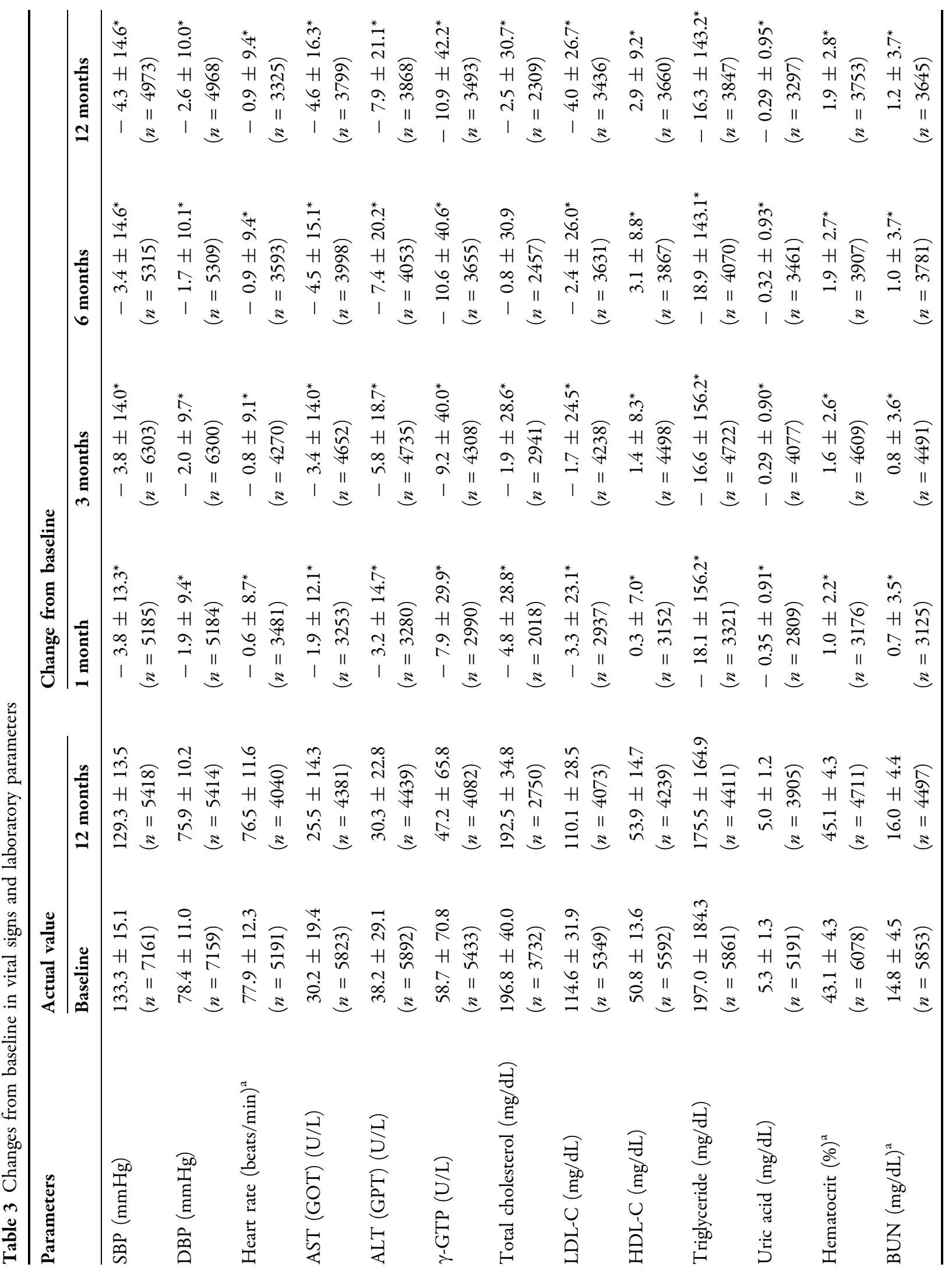




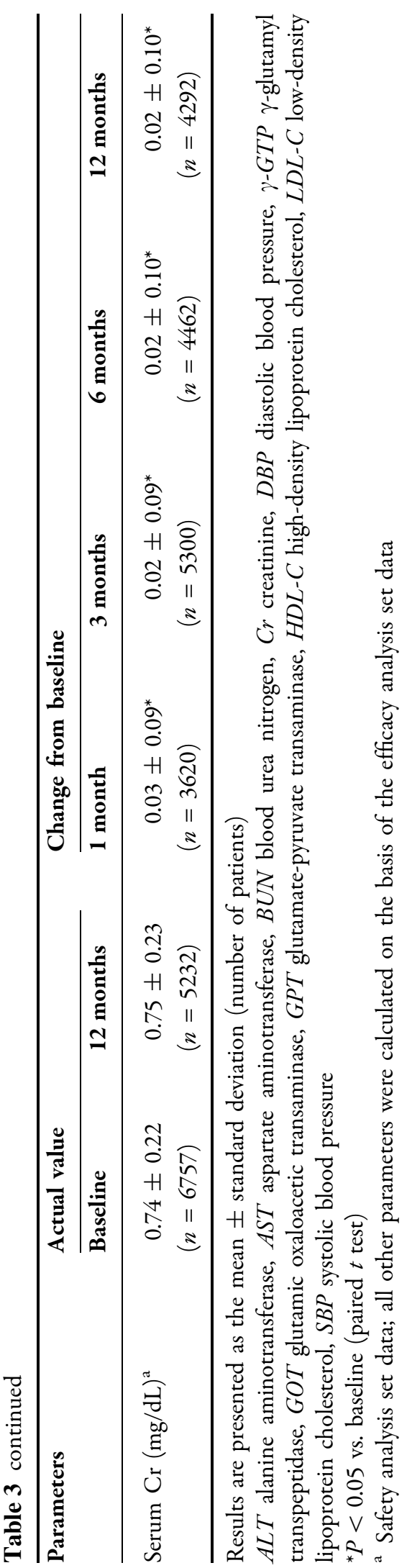

Fig. 2 Changes in eGFR (a), HbAlc (b), fasting plasma glucose (c), and body weight (d) from baseline to 12 months. HbAlc glycated hemoglobin, eGFR estimated glomerular filtration rate, SD standard deviation

which two $(0.02 \%)$ cases were considered serious. Genital infection-related ADRs included pruritus genital (40 ADRs, $0.36 \%$ ), vulvovaginal candidiasis (28 ADRs, $0.25 \%$ ), and female genital infection (20 ADRs, $0.18 \%$ ). Urinary tract infection was reported in 115 patients (1.0\%), of which five $(0.05 \%)$ cases were considered serious. ADRs associated with urinary tract infection included cystitis (56 ADRs, 0.51\%) and urinary tract infection (42 ADRs, 0.38\%). Renal disorder was reported in 115 patients $(1.0 \%)$, of which three $(0.03 \%)$ cases were considered serious. Renal disorder-related ADRs included renal disorder (27 ADRs, $0.24 \%$ ) and renal dysfunction (26 ADRs, 0.24\%). Hepatic disorder was reported in 82 patients $(0.74 \%)$, of which six $(0.05 \%)$ cases were considered serious. ADRs associated with hepatic disorder included hepatic disorder (30 ADRs, 0. 27\%) and hepatic dysfunction (28 ADRs, 0. 25\%). Hypoglycemia was reported in 39 patients $(0.35 \%)$, of which three $(0.03 \%)$ cases were considered serious. ADRs associated with hypoglycemia included hypoglycemia (32 ADRs, 0.29\%). Cardiovascular disease was reported in 30 patients $(0.27 \%)$, of which $19(0.17 \%)$ cases were considered serious. The most common ADRs associated with cardiovascular disease were acute myocardial infarction (five ADRs, 0.05\%), myocardial infarction (five ADRs, $0.05 \%$ ), angina pectoris (four ADRs, $0.04 \%$ ), unstable angina pectoris (three ADRs, 0.03\%), atrial fibrillation (three ADRs, $0.03 \%$ ), and congestive heart failure (three ADRs, 0.03\%). Cerebrovascular disease was reported in 23 patients $(0.21 \%)$, of which 20 $(0.18 \%)$ cases were considered serious. The most common ADRs associated with cerebrovascular disease were cerebral infarction (13 ADRs, $0.12 \%$ ), lacunar infarction (three ADRs, $0.03 \%$ ), transient ischemic attack (two ADRs, $0.02 \%$ ), and cerebral hemorrhage (two ADRs, $0.02 \%$ ). Malignant tumor was reported in 22 patients $(0.20 \%)$, of which $19(0.17 \%)$ cases were considered serious. ADRs associated with malignant 
(a)

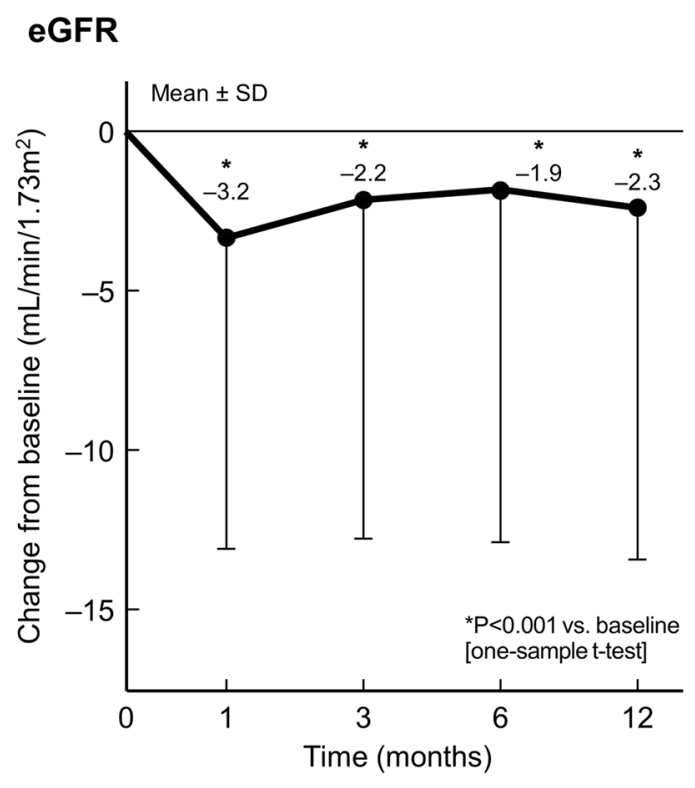

n: $3620 \quad 5300 \quad 4462$
\begin{tabular}{|c|c|}
\hline Baseline & $82.1 \pm 21.7 \mathrm{~mL} / \mathrm{min} / 1.73 \mathrm{~m}^{2}(6757)$ \\
\hline 12 months & $79.8 \pm 20.4 \mathrm{~mL} / \mathrm{min} / 1.73 \mathrm{~m}^{2}(5232)$ \\
\hline
\end{tabular}

Safety analysis set $(n=11,051)$

(c)

\section{Fasting plasma glucose}

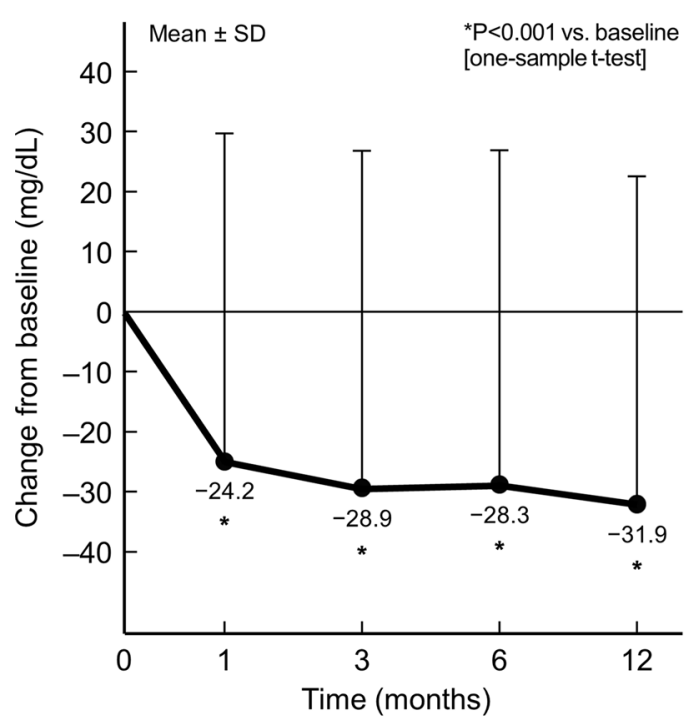

n: $3385 \quad 4302 \quad 3453 \quad 3215$
\begin{tabular}{|c|c|}
\hline Baseline & $167.1 \pm 59.8 \mathrm{mg} / \mathrm{dL}(5160)$ \\
\hline 12 months & $136.1 \pm 40.3 \mathrm{mg} / \mathrm{dL}(3708)$ \\
\hline
\end{tabular} Mean \pm SD (n) (b)

\section{HbA1c}

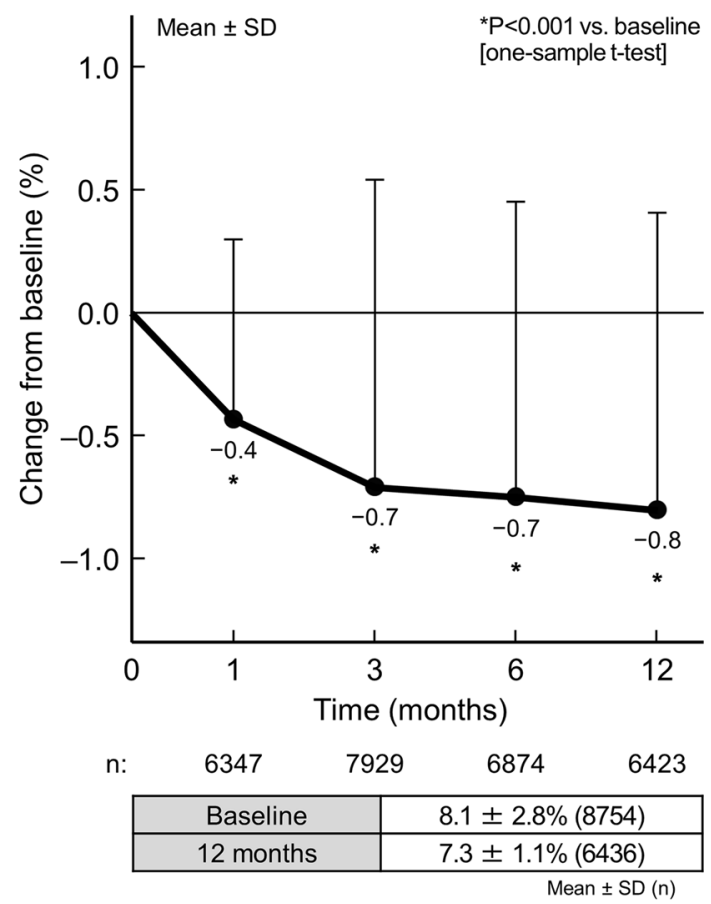

Efficacy analysis set $(n=8788)$

(d)

\section{Body weight}

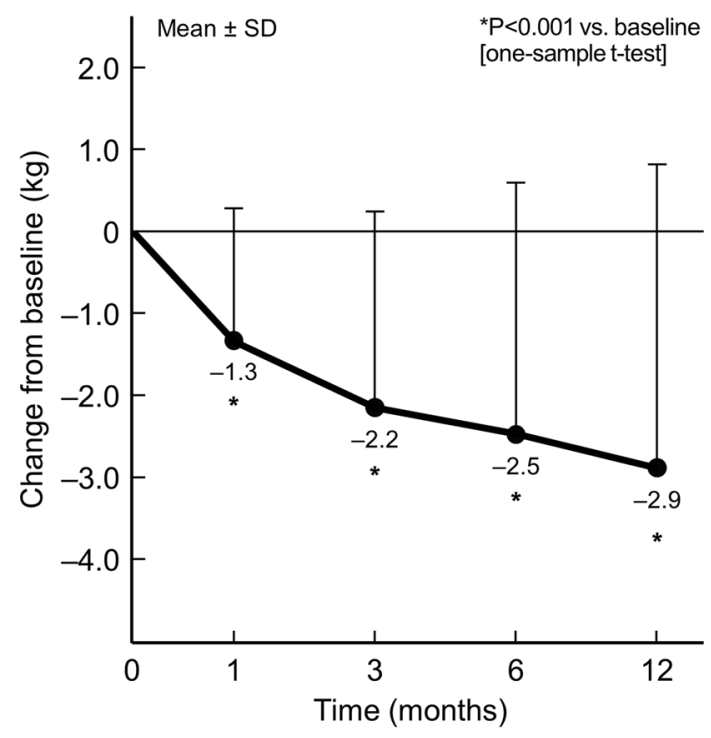

\begin{tabular}{l}
$\mathrm{n}: 4872 \quad 5976 \quad 4930 \quad 4642$ \\
\begin{tabular}{|c|r|}
\hline Baseline & $78.5 \pm 17.2 \mathrm{~kg} \mathrm{(6901)}$ \\
\hline 12 months & $75.9 \pm 16.8 \mathrm{~kg} \mathrm{(4958)}$ \\
\hline
\end{tabular} \\
\hline
\end{tabular}

Efficacy analysis set $(n=8788)$ 
Table 4 Adverse drug reactions reported during the survey period

\begin{tabular}{|c|c|c|}
\hline & $\begin{array}{l}\text { STELLA-LONG } \\
\text { TERM }\end{array}$ & $\begin{array}{l}\text { Pre-approval clinical } \\
\text { trials }\end{array}$ \\
\hline Number of patients & 11,051 & 1669 \\
\hline Number of patients with ADRs & 1616 & 549 \\
\hline Number of ADRs & 2239 & 887 \\
\hline Incidence rate $\mathrm{ADRs}$ & $14.6 \%$ & $32.9 \%$ \\
\hline \multicolumn{3}{|l|}{ System organ class } \\
\hline Infections and infestations & $201(1.8)$ & $64(3.8)$ \\
\hline $\begin{array}{l}\text { Neoplasms benign, malignant, and unspecified (incl. cysts and } \\
\text { polyps) }\end{array}$ & $25(0.23)$ & $4(0.24)$ \\
\hline Blood and lymphatic system disorders & $9(0.08)$ & $8(0.48)$ \\
\hline Immune system disorders & $1(0.01)$ & 0 \\
\hline Endocrine disorders & $1(0.01)$ & 0 \\
\hline Metabolism and nutrition disorders & $161(1.5)$ & $20(1.2)$ \\
\hline Psychiatric disorders & $9(0.08)$ & $3(0.18)$ \\
\hline Nervous system disorders & $74(0.67)$ & $42(2.5)$ \\
\hline Eye disorders & $8(0.07)$ & $17(1.0)$ \\
\hline Ear and labyrinth disorders & $1(0.01)$ & $5(0.30)$ \\
\hline Cardiac disorders & $31(0.28)$ & $10(0.60)$ \\
\hline Vascular disorders & $34(0.31)$ & $7(0.42)$ \\
\hline Respiratory, thoracic, and mediastinal disorders & $11(0.10)$ & $7(0.42)$ \\
\hline Gastrointestinal disorders & $100(0.90)$ & $101(6.1)$ \\
\hline Hepatobiliary disorders & $70(0.63)$ & $8(0.48)$ \\
\hline Skin and subcutaneous tissue disorders & $147(1.3)$ & $48(2.9)$ \\
\hline Musculoskeletal and connective tissue disorders & $23(0.21)$ & $13(0.78)$ \\
\hline Renal and urinary disorders & $644(5.8)$ & $176(10.6)$ \\
\hline Reproductive system and breast disorders & $88(0.80)$ & $25(1.5)$ \\
\hline General disorders and administration site conditions & $72(0.65)$ & $101(6.1)$ \\
\hline Investigations & $205(1.9)$ & $133(8.0)$ \\
\hline Injury, poisoning, and procedural complications & $10(0.09)$ & 0 \\
\hline
\end{tabular}

Data are presented as number of events (\%)

Coded using MedDRA/J Ver.20.1

$A D R$ adverse drug reaction, MedDRA Medical Dictionary for Regulatory Activities 
Table 5 Serious adverse drug reactions reported during the survey period

\begin{tabular}{|c|c|c|}
\hline & $\begin{array}{l}\text { STELLA-LONG } \\
\text { TERM }\end{array}$ & $\begin{array}{l}\text { Pre-approval clinical } \\
\text { trials }\end{array}$ \\
\hline Number of patients with serious ADRs & 107 & 14 \\
\hline Number of serious ADRs & 121 & 15 \\
\hline Incidence rate of serious ADRs & $0.97 \%$ & $0.84 \%$ \\
\hline \multicolumn{3}{|l|}{ System organ class } \\
\hline \multicolumn{3}{|l|}{ MedDRA preferred term } \\
\hline Infections and infestations & $9(0.08)$ & $2(0.12)$ \\
\hline Escherichia sepsis & $1(0.01)$ & 0 \\
\hline Liver abscess & $1(0.01)$ & 0 \\
\hline Peritonsillar abscess & $1(0.01)$ & 0 \\
\hline Pneumonia & $1(0.01)$ & 0 \\
\hline Pyelonephritis & 0 & $2(0.12)$ \\
\hline Pyelonephritis acute & $2(0.02)$ & 0 \\
\hline Urinary tract infection & $3(0.03)$ & 0 \\
\hline Genital herpes simplex & $1(0.01)$ & 0 \\
\hline $\begin{array}{l}\text { Neoplasms benign, malignant, and unspecified (incl. cysts and } \\
\text { polyps) }\end{array}$ & $20(0.18)$ & $3(0.18)$ \\
\hline Colon cancer & $5(0.05)$ & $1(0.06)$ \\
\hline Metastases to lymph nodes & $1(0.01)$ & 0 \\
\hline Pancreatic carcinoma & $6(0.05)$ & 0 \\
\hline Pancreatic carcinoma metastatic & $1(0.01)$ & 0 \\
\hline Squamous cell carcinoma of skin & $1(0.01)$ & 0 \\
\hline Uterine cancer & 0 & $1(0.06)$ \\
\hline Breast cancer female & $2(0.02)$ & 0 \\
\hline Lung neoplasm malignant & $1(0.01)$ & 0 \\
\hline Prostate cancer & $1(0.01)$ & $1(0.06)$ \\
\hline Lung neoplasm & $1(0.01)$ & 0 \\
\hline Thyroid cancer & $1(0.01)$ & 0 \\
\hline Intraductal proliferative breast lesion & $1(0.01)$ & 0 \\
\hline Blood and lymphatic system disorders & 0 & $1(0.06)$ \\
\hline Hemolytic anemia & 0 & $1(0.06)$ \\
\hline Endocrine disorders & $1(0.01)$ & 0 \\
\hline Hypothyroidism & $1(0.01)$ & 0 \\
\hline
\end{tabular}


Table 5 continued

\begin{tabular}{|c|c|c|}
\hline & $\begin{array}{l}\text { STELLA-LONG } \\
\text { TERM }\end{array}$ & $\begin{array}{l}\text { Pre-approval clinical } \\
\text { trials }\end{array}$ \\
\hline Metabolism and nutrition disorders & $6(0.05)$ & 0 \\
\hline Dehydration & $2(0.02)$ & 0 \\
\hline Hyperglycemia & $1(0.01)$ & 0 \\
\hline Hyperphagia & $1(0.01)$ & 0 \\
\hline Hypoglycemia & $2(0.02)$ & 0 \\
\hline Psychiatric disorders & $2(0.02)$ & 0 \\
\hline Depression & $2(0.02)$ & 0 \\
\hline Nervous system disorders & $21(0.19)$ & $1(0.06)$ \\
\hline Cerebral hemorrhage & $2(0.02)$ & 0 \\
\hline Cerebral infarction & $11(0.10)$ & $1(0.06)$ \\
\hline Depressed level of consciousness & $1(0.01)$ & 0 \\
\hline Dizziness & $1(0.01)$ & 0 \\
\hline Somnolence & $1(0.01)$ & 0 \\
\hline Transient ischemic attack & $1(0.01)$ & 0 \\
\hline Lacunar infarction & $3(0.03)$ & 0 \\
\hline Putamen hemorrhage & $1(0.01)$ & 0 \\
\hline Eye disorders & $3(0.03)$ & 0 \\
\hline Diabetic retinopathy & $1(0.01)$ & 0 \\
\hline Retinal hemorrhage & $2(0.02)$ & 0 \\
\hline Ear and labyrinth disorders & $1(0.01)$ & 0 \\
\hline Sudden hearing loss & $1(0.01)$ & 0 \\
\hline Cardiac disorders & $19(0.17)$ & $2(0.12)$ \\
\hline Acute myocardial infarction & $5(0.05)$ & $1(0.06)$ \\
\hline Angina pectoris & $2(0.02)$ & 0 \\
\hline Angina unstable & $3(0.03)$ & $1(0.06)$ \\
\hline Atrial fibrillation & $1(0.01)$ & 0 \\
\hline Cardiac failure congestive & $2(0.02)$ & 0 \\
\hline Cardiovascular disorder & $1(0.01)$ & 0 \\
\hline Coronary artery disease & $1(0.01)$ & 0 \\
\hline Myocardial infarction & $5(0.05)$ & 0 \\
\hline Vascular disorders & $3(0.03)$ & 0 \\
\hline Hypertension & $2(0.02)$ & 0 \\
\hline
\end{tabular}


Table 5 continued

\begin{tabular}{|c|c|c|}
\hline & $\begin{array}{l}\text { STELLA-LONG } \\
\text { TERM }\end{array}$ & $\begin{array}{l}\text { Pre-approval clinical } \\
\text { trials }\end{array}$ \\
\hline Varicose vein & $1(0.01)$ & 0 \\
\hline Gastrointestinal disorders & $3(0.03)$ & $1(0.06)$ \\
\hline Duodenal ulcer & $1(0.01)$ & 0 \\
\hline Gastric ulcer hemorrhage & $1(0.01)$ & 0 \\
\hline Gastroesophageal reflux disease & $1(0.01)$ & 0 \\
\hline Pancreatitis acute & $1(0.01)$ & 0 \\
\hline Upper gastrointestinal hemorrhage & 0 & $1(0.06)$ \\
\hline Hepatobiliary disorders & $7(0.06)$ & 0 \\
\hline Cholecystitis & $2(0.02)$ & 0 \\
\hline Cholecystitis acute & $1(0.01)$ & 0 \\
\hline Cholelithiasis & $1(0.01)$ & 0 \\
\hline Hepatic cirrhosis & $2(0.02)$ & 0 \\
\hline Jaundice cholestatic & $1(0.01)$ & 0 \\
\hline Liver disorder & $1(0.01)$ & 0 \\
\hline Skin and subcutaneous tissue disorders & $4(0.04)$ & 0 \\
\hline Drug eruption & $1(0.01)$ & 0 \\
\hline Eczema & $1(0.01)$ & 0 \\
\hline Skin ulcer & $1(0.01)$ & 0 \\
\hline Urticaria & $1(0.01)$ & 0 \\
\hline Renal and urinary disorders & $6(0.05)$ & $3(0.18)$ \\
\hline Hematuria & $1(0.01)$ & 0 \\
\hline Nephrolithiasis & 0 & $1(0.06)$ \\
\hline Neurogenic bladder & $1(0.01)$ & 0 \\
\hline Renal disorder & $2(0.02)$ & $1(0.06)$ \\
\hline Renal vessel disorder & $1(0.01)$ & 0 \\
\hline Ureterolithiasis & $1(0.01)$ & $1(0.06)$ \\
\hline Reproductive system and breast disorders & $1(0.01)$ & 0 \\
\hline Prostatitis & $1(0.01)$ & 0 \\
\hline General disorders and administration site conditions & $1(0.01)$ & $2(0.12)$ \\
\hline Death & 0 & $1(0.06)$ \\
\hline Pain & 0 & $1(0.06)$ \\
\hline Thirst & $1(0.01)$ & 0 \\
\hline
\end{tabular}


Table 5 continued

\begin{tabular}{lll}
\hline & $\begin{array}{l}\text { STELLA-LONG } \\
\text { TERM }\end{array}$ & $\begin{array}{l}\text { Pre-approval clinical } \\
\text { trials }\end{array}$ \\
\hline Investigations & $1(0.01)$ & 0 \\
Gamma-glutamyl transferase increased & $1(0.01)$ & 0 \\
Injury, poisoning, and procedural complications & $4(0.04)$ & 0 \\
Fall & $1(0.01)$ & 0 \\
Femoral neck fracture & $1(0.01)$ & 0 \\
Foot fracture & $1(0.01)$ & 0 \\
Ligament sprain & $1(0.01)$ & 0 \\
Road traffic accident & $1(0.01)$ & 0 \\
Subarachnoid hemorrhage & $1(0.01)$ & 0 \\
Contusion & $1(0.01)$ & 0 \\
Brain contusion & $1(0.01)$ & 0 \\
\hline
\end{tabular}

Data are presented as number of events (\%)

The numbers of patients under each system organ class (SOC) category do not necessarily add up to the total number of patients for each SOC category because some patients experienced more than one ADR

Coded using MedDRA/J Ver.20.1

$A D R$ adverse drug reaction, $M e d D R A$ Medical Dictionary for Regulatory Activities

tumors included pancreatic carcinoma (six ADRs, $0.05 \%$ ) and colon cancer (five ADRs, $0.05 \%$ ). Ketoacidosis (events related to ketonebody increase) was reported in three patients $(0.03 \%)$; none of the cases were considered serious. ADRs associated with ketoacidosis included diabetic ketoacidosis (two ADRs, $0.02 \%$ ) and ketosis (one ADR, $0.01 \%$ ). Fracture was reported in two patients $(0.02 \%)$, of which one case was considered serious (0.01\%). ADRs associated with fracture included ankle fracture, femoral neck fracture, and foot fracture (one ADR each, $0.01 \%$ each). No serious ADRs occurred in at least $1 \%$ of patients.

The time to onset of ADRs of special interest is shown in Table 7.The cumulative proportion of ADRs that occurred within 90 days from the start of treatment was $64.6 \%$ for all events (1447/2239 events), $89.4 \%$ for polyuria/pollakiuria (701/784 events), $74.7 \%$ for skin complications $\quad(127 / 170$ events), $64.3 \%$ for hypoglycemia (27/42 events), and $61.4 \%$ for genital infection (86/140 events).
ADRs of special interest according to treatment status are shown in Table 8 . The incidence rate of ADRs was highest among patients with continued treatment status $(61.0 \%)$, followed by patients with interruption or discontinuation of treatment due to an ADR (38.5\%). For all other treatment status categories, the incidence rate of ADRs was less than $4 \%$. Among patients with interruption or discontinuation of treatment due to the event, the incidence rate of skin complications was $81.3 \%$ (135/166 patients); ketoacidosis was $66.7 \%$ (2/3 patients); genital infection was $57.0 \%$ (77/135 patients); and fracture was $50 \%$ (1/2 patients). The outcomes of ADRs of special interest are shown in Table 9.

\section{Effectiveness}

The changes in HbA1c, fasting plasma glucose, and body weight from baseline to 12 months are shown in Fig. $2 b-d$, respectively. The baseline value of $\mathrm{HbA} 1 \mathrm{c}$ was $8.1 \pm 2.8 \%$ and that of fasting plasma glucose was $167.1 \pm 59.8 \mathrm{mg} / \mathrm{dL}$. The 
Table 6 Adverse drug reactions of special interest

\begin{tabular}{|c|c|c|c|c|c|c|c|}
\hline & \multicolumn{6}{|c|}{ STELLA-LONG TERM (safety analysis set $n=11,051$ ) } & \multirow{2}{*}{$\begin{array}{l}\text { Pre-approval } \\
\text { clinical trials } \\
\text { Total } \\
(n=1669)\end{array}$} \\
\hline & $\begin{array}{l}\text { Total number } \\
\text { of patients } \\
\text { experiencing } \\
\text { an ADR }\end{array}$ & & Serious & & Non-serious & & \\
\hline All ADRs & 1616 & $(14.6)$ & 107 & $(0.97)$ & 1539 & $(13.9)$ & $(32.9)$ \\
\hline \multicolumn{8}{|l|}{ ADRs of special interest } \\
\hline Polyuria/pollakiuria & 571 & $(5.2)$ & 1 & $(0.01)$ & 570 & $(5.2)$ & $(10.0)$ \\
\hline $\begin{array}{l}\text { Volume depletion-related events, } \\
\text { including dehydration }\end{array}$ & 196 & $(1.8)$ & 9 & $(0.08)$ & 189 & $(1.7)$ & $(4.5)$ \\
\hline Skin complications & 166 & $(1.5)$ & 4 & $(0.04)$ & 162 & $(1.5)$ & $(4.0)$ \\
\hline Genital infection & 135 & $(1.2)$ & 2 & $(0.02)$ & 133 & $(1.2)$ & $(2.0)$ \\
\hline Urinary tract infection & 115 & $(1.0)$ & 5 & $(0.05)$ & 110 & $(1.0)$ & $(1.8)$ \\
\hline Renal disorder & 115 & $(1.0)$ & 3 & $(0.03)$ & 112 & $(1.0)$ & $(4.8)$ \\
\hline Hepatic disorder & 82 & $(0.74)$ & 6 & $(0.05)$ & 78 & $(0.71)$ & $(1.0)$ \\
\hline Hypoglycemia & 39 & $(0.35)$ & 3 & $(0.03)$ & 36 & $(0.33)$ & $(1.4)$ \\
\hline Cardiovascular disease $^{a}$ & 30 & $(0.27)$ & 19 & $(0.17)$ & 11 & $(0.10)$ & $(1.0)$ \\
\hline Cerebrovascular disease $^{\mathrm{b}}$ & 23 & $(0.21)$ & 20 & $(0.18)$ & 3 & $(0.03)$ & $(0.2)$ \\
\hline Malignant tumor & 22 & $(0.20)$ & 19 & $(0.17)$ & 3 & $(0.03)$ & $(0.2)$ \\
\hline $\begin{array}{l}\text { Ketoacidosis, events related to } \\
\text { ketone-body increase }\end{array}$ & 3 & $(0.03)$ & 0 & $(0.00)$ & 3 & $(0.03)$ & $(1.0)$ \\
\hline Fracture & 2 & $(0.02)$ & 1 & $(0.01)$ & 1 & $(0.01)$ & 0 \\
\hline
\end{tabular}

Data are presented as number of events (\%), unless otherwise indicated

$A D R$ adverse drug reaction

${ }^{a}$ Incidence of cardiovascular disease was 4.4/1000 person-years [34] and $9.59 / 1000$ person-years [35] in the JDDM and JDCS studies, respectively

b Incidence of cerebrovascular disease was 3.1/1000 person-years [34] and 7.45/1000 person-years [35] in the JDDM and JDCS studies, respectively

improvement in glycemic control was statistically significant (both $P<0.001$ vs. baseline) at 12 months, with sustained mean reductions from baseline in HbA1c $(-0.8 \pm 1.2 \%)$ and fasting plasma glucose $(-31.9 \pm 54.7 \mathrm{mg} / \mathrm{dL})$. Statistically significant sustained reductions $(P<0.001$ vs. baseline) in body weight $(-2.9 \pm 3.7 \mathrm{~kg})$ were also observed. A significant sustained decrease $(P=0.002$ vs. baseline) in serum fasting insulin was observed, from $15.0 \pm 20.0 \mu \mathrm{U} / \mathrm{mL}$ at baseline to $12.2 \pm 10.8 \mu \mathrm{U} / \mathrm{mL}$ at 12 months (change $-4.9 \pm 22.2 \mu \mathrm{U} / \mathrm{mL})$.

\section{Liver Function}

Changes in AST and ALT from baseline to 12 months in patients with normal and abnormal liver function are shown in Fig. 3a, b, respectively. In patients with normal liver function at baseline, no clinically significant changes in AST and ALT were observed. In patients with abnormal liver function at baseline, clinically and statistically significant changes in AST $(-9.0 \pm 19.0 \mathrm{U} / \mathrm{L})$ and ALT 


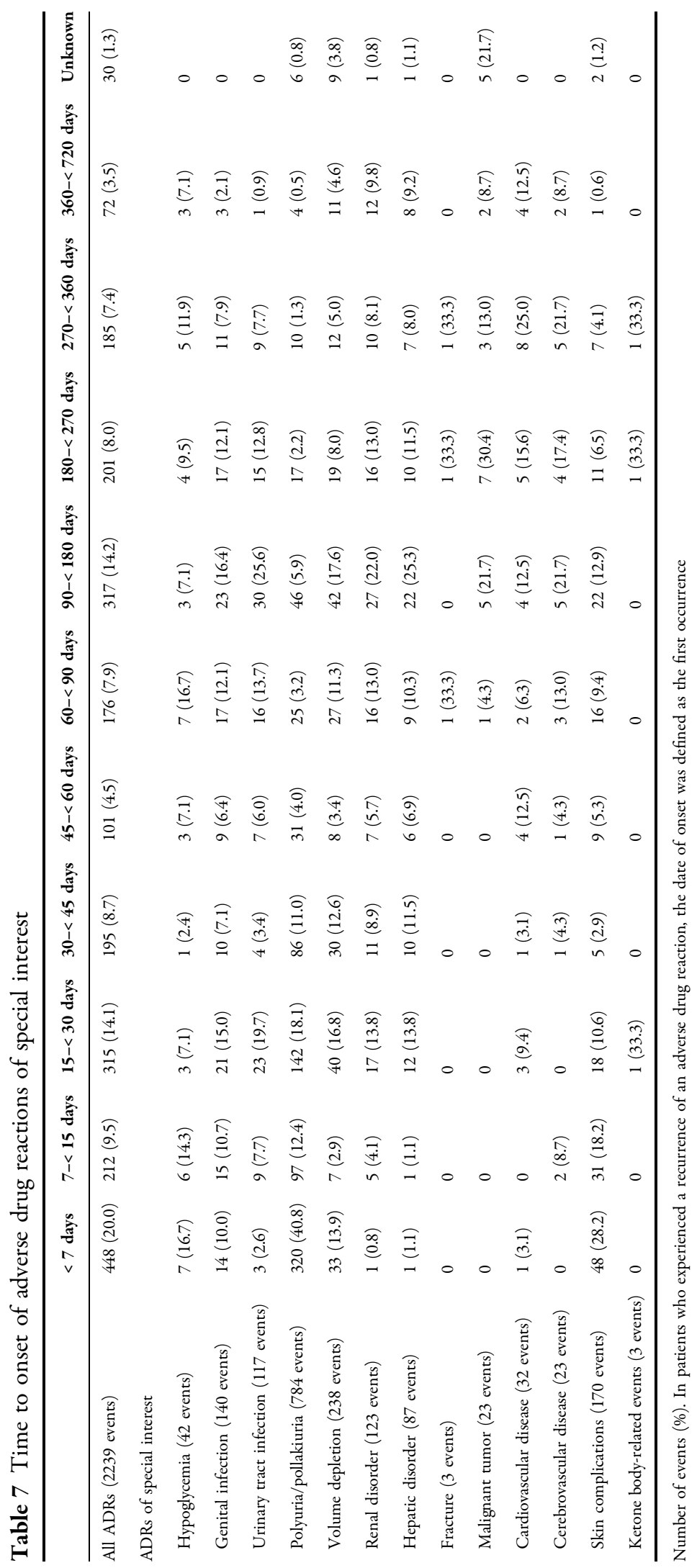




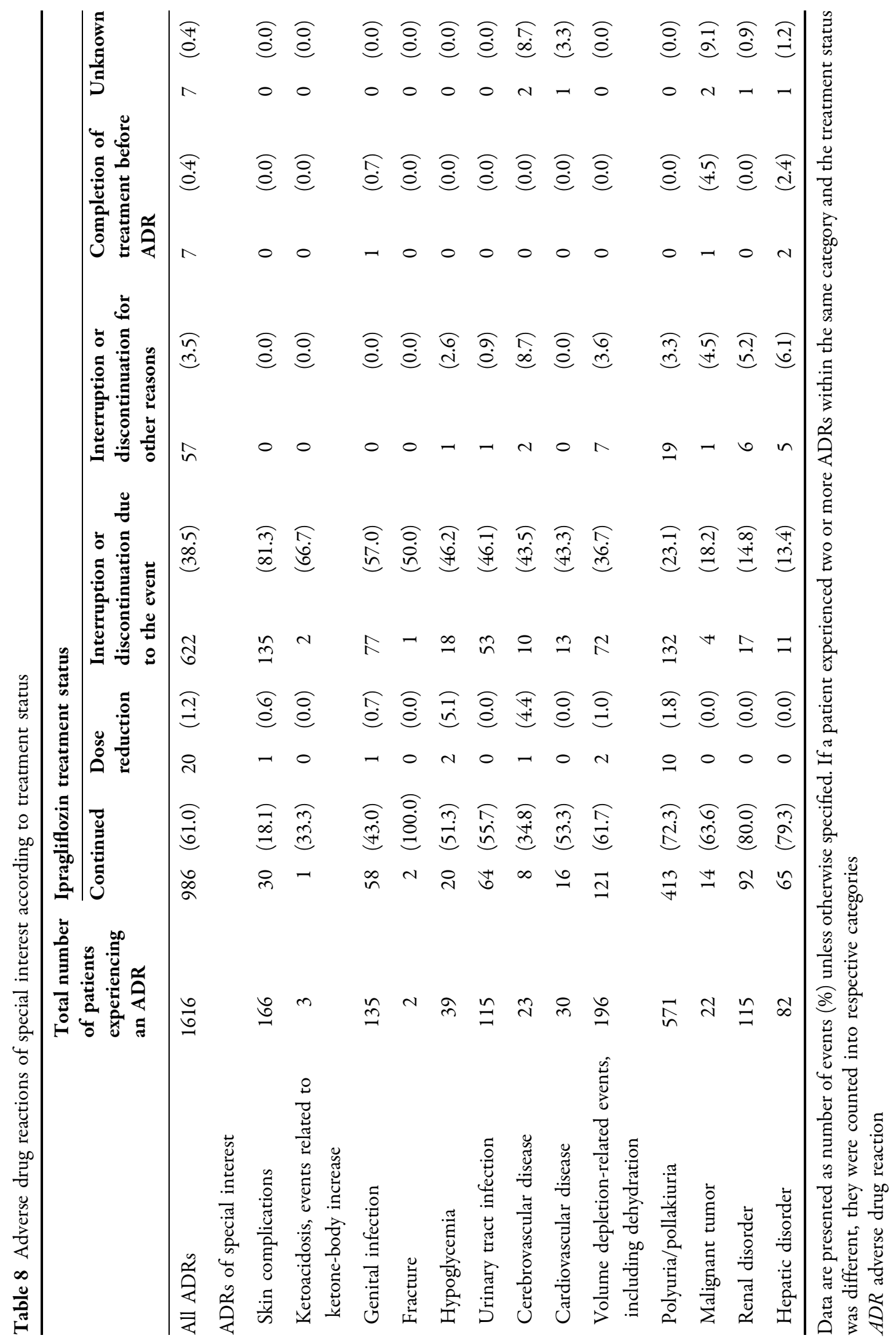


Table 9 Outcomes of adverse drug reactions of special interest

\begin{tabular}{|c|c|c|c|c|c|c|c|c|c|c|c|c|c|}
\hline \multirow[b]{3}{*}{ All ADRs } & \multirow{3}{*}{$\begin{array}{l}\text { Total } \\
2239\end{array}$} & \multicolumn{12}{|c|}{ Outcome } \\
\hline & & \multicolumn{2}{|c|}{ Resolved } & \multicolumn{2}{|c|}{ Remission } & \multicolumn{2}{|c|}{ Not recovered } & \multicolumn{2}{|c|}{ Sequelae } & \multicolumn{2}{|c|}{ Death } & \multicolumn{2}{|c|}{ Unknown } \\
\hline & & 1206 & $(53.9)$ & 707 & $(31.6)$ & 202 & $(9.0)$ & 8 & $(0.4)$ & 9 & $(0.4)$ & 107 & $(4.8)$ \\
\hline \multicolumn{14}{|l|}{ ADRs of special interest } \\
\hline Hypoglycemia & 42 & 38 & $(90.5)$ & 4 & $(9.5)$ & 0 & & 0 & & 0 & & 0 & \\
\hline Genital infection & 140 & 98 & $(70.0)$ & 33 & $(23.6)$ & 6 & $(4.3)$ & 0 & & 0 & & 3 & $(2.1)$ \\
\hline Urinary tract infection & 117 & 96 & $(82.1)$ & 19 & $(16.2)$ & 1 & $(0.9)$ & 0 & & 0 & & 1 & $(0.9)$ \\
\hline Polyuria/pollakiuria & 784 & 313 & $(39.9)$ & 353 & $(45.0)$ & 88 & $(11.2)$ & 0 & & 0 & & 30 & $(3.8)$ \\
\hline Volume depletion & 238 & 152 & $(63.9)$ & 70 & $(29.4)$ & 10 & $(4.2)$ & 1 & $(0.4)$ & 0 & & 5 & $(2.1)$ \\
\hline Renal disorder & 123 & 59 & $(48.0)$ & 31 & $(25.2)$ & 17 & $(13.8)$ & 0 & & 0 & & 16 & $(13.0)$ \\
\hline Hepatic disorder & 87 & 43 & $(49.4)$ & 12 & $(13.8)$ & 16 & $(18.4)$ & 0 & & 1 & $(1.1)$ & 15 & $(17.2)$ \\
\hline Fracture & 3 & 1 & $(33.3)$ & 2 & $(66.7)$ & 0 & & 0 & & 0 & & 0 & \\
\hline Malignant tumor & 23 & 4 & $(17.4)$ & 6 & $(26.1)$ & 3 & $(13.0)$ & 0 & & 5 & $(21.7)$ & 5 & $(21.7)$ \\
\hline Cardiovascular disease & 32 & 15 & $(46.9)$ & 11 & $(34.4)$ & 3 & $(9.4)$ & 0 & & 2 & (6.3) & 1 & $(3.1)$ \\
\hline Cerebrovascular disease & 23 & 8 & $(34.8)$ & 6 & $(26.1)$ & 0 & & 8 & $(34.8)$ & 0 & & 1 & $(4.3)$ \\
\hline Skin complications & 170 & 121 & $(71.2)$ & 42 & $(24.7)$ & 7 & $(4.1)$ & 0 & & 0 & & 0 & \\
\hline Ketone body-related events & 3 & 2 & $(66.7)$ & 1 & $(33.3)$ & 0 & & 0 & & 0 & & 0 & \\
\hline
\end{tabular}

Data are presented as number of events (\%)

$(-\quad 14.7 \pm 24.8 \mathrm{U} / \mathrm{L})$ levels were observed ( $P<0.05$ vs. baseline) .

Table 10 shows the correlation between changes in ALT and other parameters in patients with abnormal liver function. No obvious correlation was observed between the changes in ALT and changes in other parameters in patients with abnormal liver function.

Changes in the fatty liver index over time from baseline to 12 months are shown in Fig. 4 . The fatty liver index decreased significantly from $64.4 \pm 26.4$ at baseline to $55.5 \pm 27.9$ at 12 months $(-8.7 \pm 12.7, P<0.001$ vs. baseline $)$.

\section{DISCUSSION}

The STELLA-LONG TERM study is an ongoing 3-year post-marketing surveillance study. In this interim report, we present the safety and effectiveness results up to 12 months. Patient characteristics remained almost unchanged from those of the previous interim report for which $3-, 12-$, and 24-month data were pooled [4]. Other antidiabetics were concomitantly used with ipragliflozin in many patients $(81.5 \%)$. Drugs commonly used with ipragliflozin were DPP-4 inhibitors and metformin.

In the previous interim report [4], the incidence of ADRs was 10.7\% (1184/11,053). However, in the present analysis conducted on the finalized data from all patients treated for 12 months, the incidence increased to $14.6 \%$ $(1616 / 11,051)$. The incidence rates of ADRs were higher in clinical trials prior to ipragliflozin approval compared with those in the present survey for all SOC categories, except metabolism and nutrition disorders and hepatobiliary disorders.

The incidence rates of serious ADRs remained almost unchanged from those in the previous interim report [4]. There were 11 events of cerebral infarction and 6 events of pancreatic cancer, which was the most common 
(a)

AST

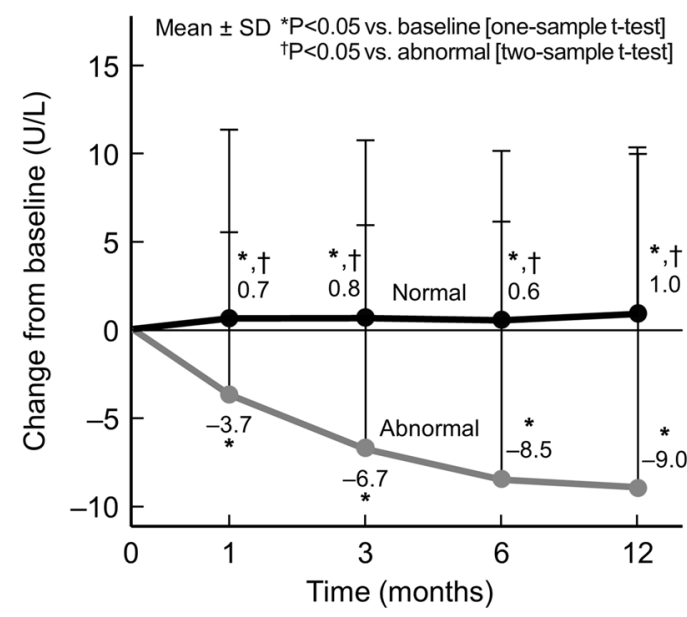

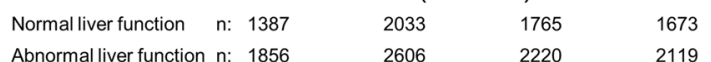

\begin{tabular}{|c|c|c|}
\hline \multirow{2}{*}{ Normal } & Baseline & $19.2 \pm 5.2 \mathrm{U} / \mathrm{L}(2566)$ \\
\cline { 2 - 3 } & 12 months & $20.2 \pm 10.1 \mathrm{U} / \mathrm{L}(1680)$ \\
\hline \multirow{2}{*}{ Abnormal } & Baseline & $38.8 \pm 21.7 \mathrm{U} / \mathrm{L}(3236)$ \\
\cline { 2 - 3 } & 12 months & $29.8 \pm 15.9 \mathrm{U} / \mathrm{L}(2130)$ \\
\hline
\end{tabular}

Efficacy analysis set $(n=8788)$ (b)

ALT

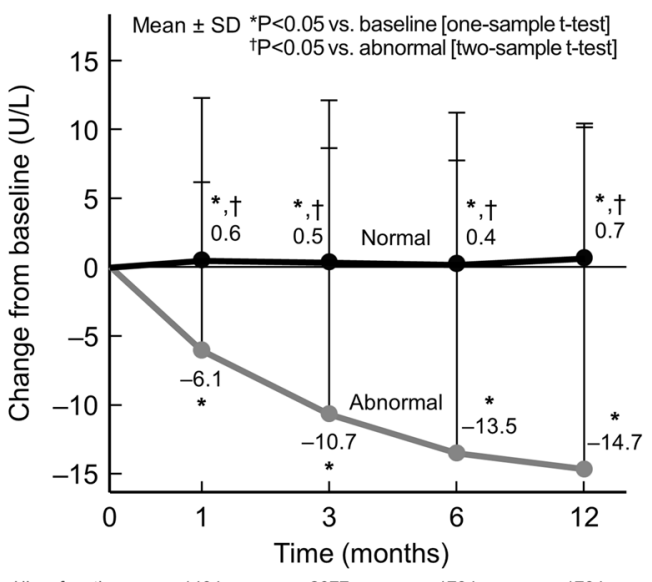

$\begin{array}{lllll}\text { Normal liver function } & \mathrm{n}: & 1404 & 2077 & 1794\end{array}$ $\begin{array}{llll}\text { Abnormal liver function n: } 1876 & 2658 & 2259 & 2164\end{array}$

\begin{tabular}{|c|c|c|}
\hline \multirow{2}{*}{ Normal } & Baseline & $18.7 \pm 5.7 \mathrm{U} / \mathrm{L}(2605)$ \\
\cline { 2 - 3 } & 12 months & $19.5 \pm 10.5 \mathrm{U} / \mathrm{L}(1704)$ \\
\hline \multirow{2}{*}{ Abnormal } & Baseline & $53.7 \pm 30.9 \mathrm{U} / \mathrm{L}(3287)$ \\
\cline { 2 - 3 } & 12 months & $38.9 \pm 25.5 \mathrm{U} / \mathrm{L}(2164)$ \\
\hline
\end{tabular}

Definition of abnormal liver function Male: ALT $\geq 31$ U/L, Female: ALT $\geq 21$ U/L

Fig. 3 Changes in AST (a) and ALT (b) from baseline to 12 months in patients stratified by liver function status. ALT alanine aminotransferase, AST aspartate aminotransferase, SD standard deviation

Table 10 Correlation between changes in ALT and other parameters in patients with abnormal liver function

\begin{tabular}{lrlr}
\hline & \multicolumn{1}{c}{$\boldsymbol{n}$} & Pearson correlation coefficient & $\boldsymbol{P}$ value \\
\hline Changes in HbAlc & 2145 & 0.149 & $<0.001$ \\
Changes in fasting plasma glucose & 1198 & 0.106 & $<0.001$ \\
Changes in fasting insulin & 103 & 0.103 & 0.302 \\
Changes in body weight & 1837 & 0.206 & $<0.001$ \\
Changes in waist circumference & 351 & 0.006 & 0.918 \\
Changes in systolic blood pressure & 1886 & 0.032 & 0.171 \\
Changes in diastolic blood pressure & 1884 & 0.081 & $<0.001$ \\
Changes in total bilirubin & 904 & 0.102 & 0.002 \\
Changes in triglycerides & 2018 & 0.050 & 0.023 \\
\hline
\end{tabular}

$A L T$ alanine aminotransferase, $H b A l c$ glycated hemoglobin

tumor type among the malignant tumors. No cases of serious ketoacidosis or lower limb amputation were observed.

The overall incidence rate of ADRs in the present interim report (14.6\%) was lower than that in clinical trials prior to ipragliflozin approval (32.9\%) [19-24]. This was also the case for individual ADRs; the incidence rate of each ADR was lower than that of clinical trials prior to ipragliflozin approval [19-24]. Genital 


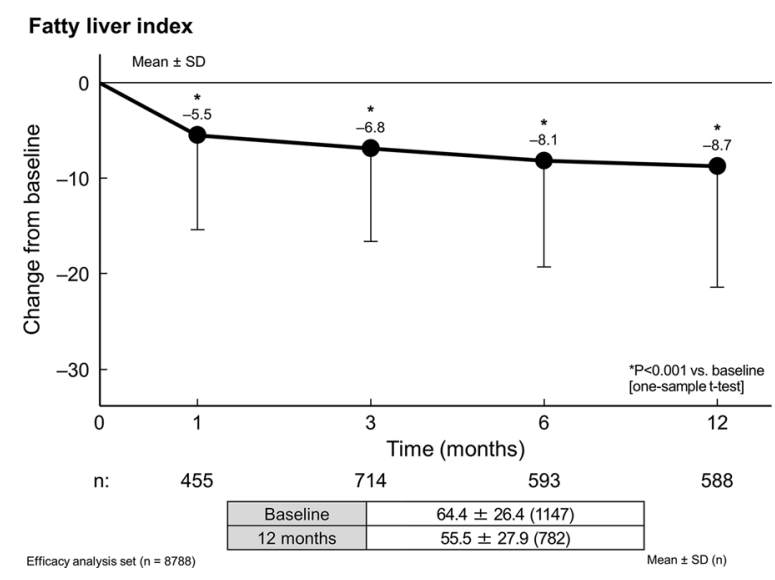

Fig. 4 Changes in fatty liver index from baseline to 12 months. SD standard deviation

infection is considered a class effect of SGLT2 inhibitors, although a recent meta-analysis reported that rates of genital mycotic infection were significantly higher in patients who received daily canagliflozin (100 or $300 \mathrm{mg}$ ) vs sitagliptin (100 mg), a DPP-4 inhibitor [25]. In the present study, 28 ADRs of vulvovaginal candidiasis were reported $(0.25 \%)$, though it remains to be determined whether this is comparable with other SGLT2 inhibitors.

Regarding the time to onset of ADRs, approximately $65 \%$ of all ADRs occurred within 90 days of the start of the medication. Nearly $90 \%$ of polyuria/pollakiuria events and nearly $75 \%$ of skin complications occurred within 90 days. There was no particular trend in the onset time of fractures or malignant tumors.

In the present interim report, we investigated ADRs of special interest according to treatment status. In $81.3 \%$ of patients who experienced skin complications, treatment was interrupted or discontinued because of the event. This finding is likely related to the recommendations issued by experts advising caution with the use of SGLT2 inhibitors, especially in regard to skin problems [26].

Most ADRs resolved in the majority of patients. However, this did not apply to cases of malignant tumors and cerebrovascular/cardiovascular diseases. Regarding the incidence rate of ADRs by patient demographic and clinical characteristics, the incidences of ADRs were high, mainly in patients with long duration of disease, patients receiving increased doses of ipragliflozin, patients using many concomitant medications, and patients with higher baseline HbA1c levels.

The extent of decreases in $\mathrm{HbA} 1 \mathrm{c}$ and fasting plasma glucose levels observed in the early phase of treatment were maintained for 12 months. The low rate of discontinuations due to "no improvement or worsening" (3.5\%) and the use of concomitant medications and/or optimal dose modifications under the close supervision of the attending physician may be attributed to the favorable glycemic control.

Although statistically significant changes from baseline to 12 months in vital signs and all other laboratory parameters were observed, some of these changes may not be clinically significant and may have resulted from the large sample size. Favorable changes in blood pressure, lipids, plasma glucose, and uric acid suggest that a favorable effect on cardiovascular event risk may be expected, although this remains to be confirmed when the 3-year data become available. Statistically significant reductions from baseline to 12 months in AST and ALT were observed for patients with abnormal liver function status at baseline, while no clinically significant changes were shown in patients with normal liver function. This finding is consistent with that observed in a previous interim report of liver function of the STELLA-LONG TERM study [6]. Previous studies on other SGLT2 inhibitors have also reported an improvement in liver function-related parameters (AST and ALT) [27-30]. Taken together, these findings suggest that T2DM patients, especially those with abnormal liver function, may benefit from treatment with ipragliflozin.

No obvious correlation between ALT and change in each parameter was observed. This finding is consistent with that of a previous study on another SGLT2 inhibitor in which no correlation was found between HbA1c improvement and liver function [30].

The improvement in fatty liver index observed in the present study was consistent with that shown previously [31]. The fatty liver index is a surrogate marker of hepatic steatosis, which has been suggested to have a prognostic value for the risk of diabetes mellitus [32]. 
Future research on liver function will focus on the association between changes in fatty liver index values and other parameters and on comparing abnormal and normal fatty liver index subgroups.

The present study has some limitations. There was potential bias from incorrect completion of the survey report forms, which may have led to over- or under-representation of ADRs. The absence of a control group for comparison raises the possibility that the incidence of ADRs, as well as improvements in effectiveness variables, may be attributed to factors other than ipragliflozin (e.g., concomitant medications).

\section{CONCLUSION}

Ipragliflozin was effective and well tolerated in Japanese patients with T2DM over 12 months in a real-world clinical setting. Improvements in liver function parameters (AST and ALT) were observed in T2DM patients with abnormal liver function. The results reported here should be considered preliminary in nature and the results of further analyses are planned to be published as the data become available in the future.

\section{ACKNOWLEDGEMENTS}

We would like to thank the study investigators and participating patients.

Funding. This study was sponsored by Astellas Pharma Inc., Japan. Astellas Pharma Inc., Japan also provided funding for the article processing charges and open access fees. All authors had full access to all of the data in this study and take complete responsibility for the integrity of the data and accuracy of the data analysis.

Medical Writing and Editorial Assistance. Medical writing and editorial support was funded by Astellas and provided by Dr. Michelle Belanger (Edanz Medical Writing) and EMC K.K.

Authorship. All named authors meet the International Committee of Medical Journal Editors (ICMJE) criteria for authorship for this article, take responsibility for the integrity of the work as a whole, and have given their approval for this version to be published.

Prior Presentation. This manuscript has not been published elsewhere and is not under consideration in whole or in part by another journal, except as an oral presentation at the 61st Annual Meeting of the Japan Diabetes Society (2018).

Disclosures. Ichiro Nakamura is an employee of Astellas Pharma Inc. Hiroshi Maegawa has received lecture fees from Astellas Pharma Inc., MSD K.K., Daiichi Sankyo Company Limited, Nippon Boehringer Ingelheim Co., Ltd., Kissei Pharmaceutical Co., Mitsubishi Tanabe Pharma Corporation, Sanofi K.K., Kowa Pharmaceutical Company Ltd., and Takeda Pharmaceutical Company Limited; research support from Astellas Pharma Inc., AstraZeneca K.K., and Boehringer Ingelheim Co., Ltd.; and grants from Takeda Pharmaceutical Company Limited, Astellas Pharma Inc., MSD K.K., Teijin Limited, Nippon Boehringer Ingelheim Co., Ltd., Kyowa Hakko Kirin Co., Ltd., Taisho Toyama Pharmaceutical Co., Ltd., Kowa Pharmaceutical Company Ltd., Ono Pharmaceutical Co., Ltd., Daiichi Sankyo Company Limited, Sanofi K.K., Mitsubishi Tanabe Pharma Corporation, Shionogi \& Co., Ltd., Otsuka Pharmaceutical Co., Ltd., and Sanwa Kagaku Kenkyusho Co., Ltd. Kazuyuki Tobe has received lecture fees from Astellas Pharma Inc., AstraZeneca K.K., Kowa Pharmaceutical Company Ltd., Takeda Pharmaceutical Company Limited, and Novo Nordisk Pharma Ltd.; and grants from Kowa Pharmaceutical Company Ltd., Astellas Pharma Inc., Ono Pharmaceutical Co., Ltd., Mitsubishi Tanabe Pharma Corporation, Daiichi Sankyo Company Limited, Nippon Boehringer Ingelheim Co., Ltd., Takeda Pharmaceutical Company Limited, Sanofi K.K., and Fuji Chemical Industries Co., Ltd. Satoshi Uno is an employee of Astellas Pharma Inc.

Compliance with Ethics Guidelines. This post-marketing surveillance study was in compliance with Good Post-marketing Study Practice. This study involved the collection of 
anonymized data from a clinical setting as required by the regulatory body; therefore, informed consent was waived in compliance with the Japanese regulations for post-marketing surveillance studies. All of the medical institutions that agreed to provide data signed a contract with Astellas Pharma Inc.

Open Access. This article is distributed under the terms of the Creative Commons Attribution-NonCommercial 4.0 International License (http://creativecommons.org/licenses/ by-nc/4.0/), which permits any noncommercial use, distribution, and reproduction in any medium, provided you give appropriate credit to the original author(s) and the source, provide a link to the Creative Commons license, and indicate if changes were made.

\section{REFERENCES}

1. Poole RM, Dungo RT. Ipragliflozin: first global approval. Drugs. 2014;74:611-7.

2. Abdul-Ghani MA, Norton L, DeFronzo RA. Efficacy and safety of SGLT2 inhibitors in the treatment of type 2 diabetes mellitus. Curr Diab Rep. 2012;12:230-8.

3. Maegawa $H$, Tobe $K$, Tabuchi $H$, Nakamura I. Baseline characteristics and interim (3-month) efficacy and safety data from STELLA-LONG TERM, a long-term post-marketing surveillance study of ipragliflozin in Japanese patients with type 2 diabetes in real-world clinical practice. Expert Opin Pharmacother. 2016;17:1985-94.

4. Nakamura I, Maegawa H, Tobe K, Tabuchi H, Uno S. Safety and efficacy of ipragliflozin in Japanese patients with type 2 diabetes in real-world clinical practice: interim results of the STELLA-LONG TERM post-marketing surveillance study. Expert Opin Pharmacother. 2018;19:189-201.

5. Maegawa H, Tobe K, Tabuchi H, Nakamura I, Uno S. Safety and efficacy of ipragliflozin in elderly versus non-elderly Japanese patients with type 2 diabetes mellitus: a subgroup analysis of the STELLA-LONG TERM study. Expert Opin Pharmacother. 2018;19:327-36.

6. Tabuchi H, Maegawa H, Tobe K, Nakamura I, Uno S. Effect of ipragliflozin on liver function in Japanese type 2 diabetes mellitus patients: a subgroup analysis of the STELLA-LONG TERM study (3month interim results). Endocr J. 2018. https://doi. org/10.1507/endocrj.ej18-0217.

7. Nishimura N, Kitade $M$, Noguchi R, et al. Ipragliflozin, a sodium-glucose cotransporter 2 inhibitor, ameliorates the development of liver fibrosis in diabetic Otsuka Long-Evans Tokushima fatty rats. J Gastroenterol. 2016;51:1141-9.

8. Honda Y, Imajo K, Kato T, et al. The selective SGLT2 inhibitor ipragliflozin has a therapeutic effect on nonalcoholic steatohepatitis in mice. PLoS One. 2016;11:e0146337.

9. Uchida J, Kabeya Y, Oikawa Y, et al. The ameliorating effect of ipragliflozin, a SGLT2 inhobitor, on liver function without dependence on the body weight reduction. J Jpn Diabetes Soc. 2016;59:782-90.

10. Jojima T, Tomotsune T, Iijima T, Akimoto K, Suzuki $\mathrm{K}$, Aso Y. Empagliflozin (an SGLT2 inhibitor), alone or in combination with linagliptin (a DPP-4 inhibitor), prevents steatohepatitis in a novel mouse model of non-alcoholic steatohepatitis and diabetes. Diabetol Metab Syndr. 2016;8:45.

11. Komiya C, Tsuchiya K, Shiba K, et al. Ipragliflozin Improves hepatic steatosis in obese mice and liver dysfunction in type 2 diabetic patients irrespective of body weight reduction. PLoS One. 2016;11:e0151511.

12. Hayashizaki-Someya Y, Kurosaki E, Takasu T, et al. Ipragliflozin, an SGLT2 inhibitor, exhibits a prophylactic effect on hepatic steatosis and fibrosis induced by choline-deficient L-amino acid-defined diet in rats. Eur J Pharmacol. 2015;754:19-24.

13. Qiang S, Nakatsu Y, Seno Y, et al. Treatment with the SGLT2 inhibitor luseogliflozin improves nonalcoholic steatohepatitis in a rodent model with diabetes mellitus. Diabetol Metab Syndr. 2015;7:104.

14. Yokono M, Takasu T, Hayashizaki Y, et al. SGLT2 selective inhibitor ipragliflozin reduces body fat mass by increasing fatty acid oxidation in high-fat diet-induced obese rats. Eur J Pharmacol. 2014;727:66-74.

15. Suzuki M, Takeda M, Kito A, et al. Tofogliflozin, a sodium/glucose cotransporter 2 inhibitor, attenuates body weight gain and fat accumulation in diabetic and obese animal models. Nutr Diabetes. 2014;4:e125.

16. Tahara A, Kurosaki E, Yokono M, et al. Effects of SGLT2 selective inhibitor ipragliflozin on hyperglycemia, hyperlipidemia, hepatic steatosis, 
oxidative stress, inflammation, and obesity in type 2 diabetic mice. Eur J Pharmacol. 2013;715:246-55.

17. Kashiwagi A, Yoshida S, Nakamura I, et al. Efficacy and safety of ipragliflozin in Japanese patients with type 2 diabetes stratified by body mass index: a subgroup analysis of five randomized clinical trials. J Diabetes Investig. 2016;7:544-54.

18. Bedogni G, Bellentani S, Miglioli L, et al. The Fatty Liver Index: a simple and accurate predictor of hepatic steatosis in the general population. BMC Gastroenterol. 2006;6:33.

19. Kadokura T, Akiyama N, Kashiwagi A, et al. Pharmacokinetic and pharmacodynamic study of ipragliflozin in Japanese patients with type 2 diabetes mellitus: a randomized, double-blind, placebocontrolled study. Diabetes Res Clin Pract. 2014;106:50-6.

20. Kashiwagi A, Akiyama N, Shiga T, et al. Efficacy and safety of ipragliflozin as an add-on to a sulfonylurea in Japanese patients with inadequately controlled type 2 diabetes: results of the randomized, placebocontrolled, double-blind, phase III EMIT study. Diabetol Int. 2015;6:125-38.

21. Kashiwagi A, Kazuta K, Goto K, et al. Ipragliflozin in combination with metformin for the treatment of Japanese patients with type 2 diabetes: ILLUMINATE, a randomized, double-blind, placebo-controlled study. Diabetes Obes Metab. 2015;17:304-8.

22. Kashiwagi A, Kazuta K, Takinami Y, Yoshida S, Utsuno A, Nagase I. Ipragliflozin improves glycemic control in Japanese patients with type 2 diabetes mellitus: the BRIGHTEN study. Diabetol Int. $2015 ; 6: 8-18$.

23. Kashiwagi A, Shiga T, Akiyama N, et al. Efficacy and safety of ipragliflozin as an add-on to pioglitazone in Japanese patients with inadequately controlled type 2 diabetes: a randomized, double-blind, placebo-controlled study (the SPOTLIGHT study). Diabetol Int. 2015;6:104-16.

24. Kashiwagi A, Takahashi $\mathrm{H}$, Ishikawa $\mathrm{H}$, et al. A randomized, double-blind, placebo-controlled study on long-term efficacy and safety of ipragliflozin treatment in patients with type 2 diabetes mellitus and renal impairment: results of the longterm ASP1941 safety evaluation in patients with type 2 diabetes with renal impairment (LANTERN) study. Diabetes Obes Metab. 2015;17:152-60.

25. Bundhun PK, Huang F. Adverse drug events associated with sitagliptin versus canagliflozin for the treatment of patients with type 2 diabetes mellitus: a systematic comparison through a meta-analysis. Diabetes Ther. 2018;9:1883-95.
26. Yabe D, Nishikino R, Kaneko M, et al. Short-term impacts of sodium/glucose co-transporter 2 inhibitors in Japanese clinical practice: considerations for their appropriate use to avoid serious adverse events. Expert Opin Drug Saf. 2015;14:795-800.

27. Tobita H, Sato S, Miyake T, Ishihara S, Kinoshita Y. Effects of dapagliflozin on body composition and liver tests in patients with nonalcoholic steatohepatitis associated with type 2 diabetes mellitus: a prospective, open-label, uncontrolled study. Curr Ther Res Clin Exp. 2017;87:13-9.

28. Seko Y, Sumida Y, Sasaki K, et al. Effects of canagliflozin, an SGLT2 inhibitor, on hepatic function in Japanese patients with type 2 diabetes mellitus: pooled and subgroup analyses of clinical trials. J Gastroenterol. 2018;53:140-51.

29. Katsuyama H, Hamasaki $H$, Adachi $H$, et al. Effects of sodium-glucose cotransporter 2 inhibitors on metabolic parameters in patients with type 2 diabetes: a chart-based analysis. J Clin Med Res. 2016;8:237-43.

30. Kuchay MS, Krishan S, Mishra SK, et al. Effect of empagliflozin on liver fat in patients with type 2 diabetes and nonalcoholic fatty liver disease: a randomized controlled trial (E-LIFT Trial). Diabetes Care. 2018;41:1801-8.

31. Takase T, Nakamura A, Miyoshi H, Yamamoto C, Atsumi T. Amelioration of fatty liver index in patients with type 2 diabetes on ipragliflozin: an association with glucose-lowering effects. Endocr J. 2017;64:363-7.

32. Yadav D, Choi E, Ahn SV, et al. Fatty liver index as a simple predictor of incident diabetes from the KoGESARIRANG study. Med (Baltimore). 2016;95:e4447.

33. Ministry of Health, Labour and Welfare. Classification criteria for seriousness of adverse drug reactions of pharmaceuticals. PAB/SD notification no. 80. Tokyo: Ministry of Health, Labour and Welfare, 1992.

34. Yokoyama H, Matsushima M, Kawai K, et al. Low incidence of cardiovascular events in Japanese patients with type 2 diabetes in primary care settings: a prospective cohort study (JDDM 20). Diabet Med. 2011;28:1221-8.

35. Sone $H$, Tanaka $S$, Tanaka $S$, et al. Serum level of triglycerides is a potent risk factor comparable to LDL cholesterol for coronary heart disease in Japanese patients with type 2 diabetes: subanalysis of the Japan Diabetes Complications Study (JDCS). J Clin Endocrinol Metab. 2011;96:3448-56. 